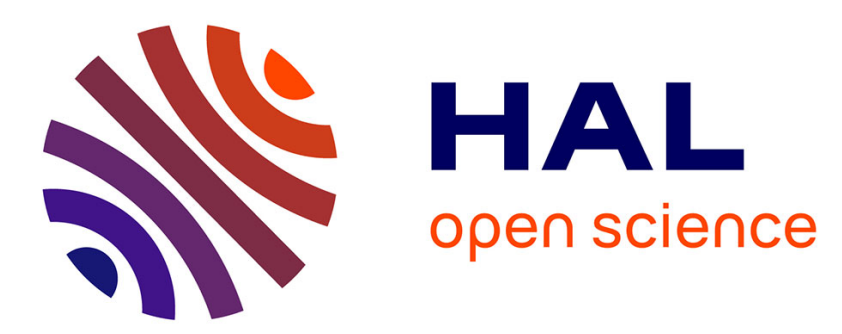

\title{
Une sous-famille caractéristique des forêts humides primaires malgaches: les Rossodinae (Trichoptera, Philopotamidae)
}

François-Marie Gibon

\section{- To cite this version:}

François-Marie Gibon. Une sous-famille caractéristique des forêts humides primaires malgaches: les Rossodinae (Trichoptera, Philopotamidae). Zoosystema, 2013, 35 (2), pp.151 - 174. 10.5252/z2013n2a2 . hal-01218261

\section{HAL Id: hal-01218261 \\ https://hal.science/hal-01218261}

Submitted on 20 Oct 2015

HAL is a multi-disciplinary open access archive for the deposit and dissemination of scientific research documents, whether they are published or not. The documents may come from teaching and research institutions in France or abroad, or from public or private research centers.
L'archive ouverte pluridisciplinaire HAL, est destinée au dépôt et à la diffusion de documents scientifiques de niveau recherche, publiés ou non, émanant des établissements d'enseignement et de recherche français ou étrangers, des laboratoires publics ou privés. 


\title{
Une sous-famille caractéristique des forêts humides primaires malgaches : les Rossodinae (Trichoptera, Philopotamidae)
}

\author{
François-Marie GIBON \\ Centre de Biologie pour la Gestion des Populations \\ (UMR INRA/IRD/CIRAD/Montpellier Supagro) \\ Campus de Baillarguet, CS 30016 , \\ F-34988 Montferrier sur Lez Cedex (France) \\ francois-marie.gibon@ird.fr
}

\footnotetext{
MOTS CLÉS

Trichoptera, Philopotamidae,

Rossodinae,

Madagascar,

biogéographie,

espèces nouvelles.
}

Gibon F.-M. 2013. - Une sous-famille caractéristique des forêts humides primaires malgaches : les Rossodinae (Trichoptera, Philopotamidae). Zoosystema 35 (2): 151-174. http://dx.doi. org/10.5252/z2013n2a2

\section{RÉSUMÉ}

Les Rossodinae Özdikmen \& Darilmaz, 2008 sont l'une des trois sous-familles constitutives des Philopotamidae Stephens, 1829. Ils ne sont connus que par deux spécimens-types provenant du massif du Tsaratanana. Un inventaire de la faune aquatique des eaux douces malgaches a permis la découverte d'un matériel plus abondant qui comprend 15 espèces, toutes nouvelles et décrites dans ce travail : Rossodes mantadia n. sp., $R$. pilakai n. sp., $R$. hertui n. sp., $R$. goodmani n. sp., $R$. manantenina n. sp., $R$. fabienneae n. sp., $R$. andohahela n. sp., $R$. ambreensis n. sp., $R$. namorona n. sp., $R$. marojejyensis $\mathrm{n}$. sp., $R$. langrandi n. sp., $R$. humbert $i$ n. sp., $R$. rakotonirinai $\mathrm{n}$. sp., $R$. ambatomisana n. sp. et $R$. ankaratra n. sp. L'étude morphologique confirme la monophylie de la lignée et atteste son ancienneté. Les espèces se distinguent entre elles par des caractères des genitalia mâles, en particulier le dixième segment abdominal, les appendices supérieurs et les structures internes de l'appareil phallique. La modification des appendices inférieurs, un caractère apomorphe, est commun à toutes les espèces. En revanche, la présence de la furca 4 aux ailes postérieures du mâle, un des caractères les plus plésimorphe, n’a pas été observée chez les nouvelles espèces et n'existe que chez l'espèce du Tsaratanana. Le genre est présent du nord au sud de Madagascar, depuis le niveau de la mer jusqu'à une altitude de $1800 \mathrm{~m}$. Il est strictement cantonné à de petits ruisseaux des forêts humides primaires. Sa distribution est limitée au versant oriental et à certaines reliques forestières des Hautes-Terres Centrales. Ce groupe illustre l'effet déterminant de la végétation sur les conditions physiques et chimiques des eaux courantes et, en conséquence, la faune aquatique. Les répartitions géographiques de toutes les espèces sont réduites, généralement limitées à un seul bassin versant. 


\section{KEY WORDS}

Trichoptera,

Philopotamidae,

Rossodinae,

Madagascar,

biogeography, ecology,

new species.
ABSTRACT
Rossodinae, a subfamily characteristic of the pristine Malagasy rainforests (Trichoptera,
Philopotamidae).
The Rossodinae Özdikmen \& Darilmaz, 2008, one of the three sub-families of Philopotamidae Stephens, 1829 are only known by two type specimens from the Tsaratanana Mountain, from which this subfamily has been described. An inventory of the Malagasy aquatic fauna has resulted in the discovery of a more abundant material that includes 15 species, all new and described here: Rossodes mantadia n. sp., $R$. pilakai n. sp., $R$. hertui n. sp., $R$. goodmani n. sp., $R$. manantenina n. sp., $R$. fabienneae n. sp., $R$. andohahela n. sp., $R$. ambreensis n. sp., $R$. namorona n. sp., $R$. marojejyiensis, $R$. langrandi n. sp., $R$. humberti n. sp., $R$. rakotonirinai n. sp., $R$. ambatomisana n. sp. and $R$. ankaratra n. sp. The morphological study confirmed the monophyly and the antiquity of the lineage. One of the plesiomorphic characters, the furca 4 of the male hindwings, has not been observed among the new species and exist only in the species originating from the Tsaratanana. The deformation of the inferior appendages, a remarkable apomorphy, is present in the whole lineage. Characters of male genitalia distinguish the different species, particularly the tenth tergite, the superior appendages and the internal sclerites of the phallic apparatus. The genus occurs from North to South of the island, from sea level up to $1800 \mathrm{~m}$. It is strictly confined to small creeks in humid pristine forests. Its distribution is limited to the eastern slope and some of the last forests in the Central Highlands. This group illustrates the decisive effect of the vegetation on the physical and chemical conditions of running waters and, as a consequence, on aquatic wildlife. The specific distributions are small, generally limited to a single watershed.

\section{INTRODUCTION}

Les Philopotamidae Stephens, 1829 sont répartis en trois sous-familles : Philopotaminae Stephens, 1829, Chimarrinae Rambur, 1842 et Rossodinae Özdikmen \& Darilmaz, 2008. Les Chimarrinae ne comprennent que trois genres Chimarra Stephens, 1829, Chimarrhodella Lestage, 1925 et Edidiehlia Malicky, 1993. Edidiehlia est un genre asiatique monospécifique, Chimarrhodella un genre néotropical comprenant peu d'espèces (12); en revanche, Chimarra est un genre cosmopolite et diversifié, présent et souvent abondant dans la plupart des eaux courantes; il comprend plus de 600 espèces sans compter une soixantaine de formes malgaches en cours d'étude (Gibon 2001). Les Philopotaminae comprennent 18 genres; ils sont présents dans toutes les régions biogéographiques à l'exception de l'Antarctique et de certaines îles océaniques. Ils sont cependant peu représentés dans les régions afrotropicale et malgache ; une douzaine d'espèces seulement y sont décrites dont quatre de Madagascar. La sous-famille des Rossodinae, endémique malgache, était jusqu'à présent monospécifique. Elle a été décrite par Ross (1956), sous l'appellation de Paulianodinae Ross, 1956, à partir d'un mâle et d'une femelle capturés sur le massif du Tsaratanana (Anonyme 1970). Dans la même publication, Ross décrit le genre Paulianodes et l'espèce $P$. tsaratananae. Özdikmen et Darilmaz (2008), constatant que le 
nom Paulianodes avait déjà été utilisé par Mamet (1954) pour un genre d'Hémiptères, ont proposé respectivement les noms de Rossodinae, Rossodes et Rossodes tsaratananae (Ross, 1956) en application de l'article $60 \mathrm{du}$ Code de Nomenclature Zoologique.

Au cours du programme "Biodiversité et Biotypologie des eaux continentales malgaches» mené en coopération par l'Institut français de Recherche pour le Développement en coopération (IRD) et le Centre National de Recherches sur l'Environnement, nous avons mis en évidence la présence du genre Rossodes dans d'autres régions de l'Est et du centre de Madagascar (Gibon 2000, 2001, 2004). Cet inventaire de plus de 400 stations, réparties en fonction de la variété des cours d'eau et des milieux offerte par la Grande Île, a permis la récolte de 15 espèces, toutes distinctes de $R$. tsaratananae. Le fait que nous n'ayons pu échantillonner à nouveau la région du Tsaratanana explique l'absence de nouvelle capture de l'espèce type. Les nouvelles espèces, décrites ci-dessous, permettent l'évaluation des caractères utilisés pour créer la sous-famille. Enfin, nous présentons une description des conditions écologiques où se rencontrent les Rossodes, ainsi qu'une analyse de leurs répartitions qui jettent un éclairage particulier sur l'exceptionnelle richesse biologique malgache.

\section{MATÉRIEL ET MÉTHODES}

L'ensemble du projet qui a permis ces travaux est décrit en détail par Elouard \& Gibon (2001). Dans les parcs nationaux, où l'accès à certains sites nécessitait plusieurs jours de marche, les missions ont été organisées en collaboration avec le WWF. La présentation des milieux naturels ainsi qu'un premier bilan des inventaires biologiques ont alors été publiés (Parc national de l'Andringitra : Goodman 1996 ; Réserve Spéciale d'Anjanaharibe sud : Goodman 1998 ; Parc national de l'Andohahela : Goodman 1999 ; Parc national du Marojejy: Goodman 2000).

Les spécimens ont été capturés à l'aide d'un piège lumineux portable composé d'une source de lumière noire et d'une lampe à gaz. Ils sont conservés dans de l'éthanol à $75 \%$. Les genitalia ont été éclaircis dans une solution d'hydroxyde de potassium, étudiés au microscope dans de l'essence de girofle (ou de cèdre) puis montés sur lame dans de l'euparal. Les holotypes sont déposés au Muséum national d'Histoire naturelle, Paris (MNHN) n 1488 . Le reste du matériel est dans la collection de l'auteur, déposée au Centre de Biologie pour la Gestion des Populations (CBGP, France Campus International de Baillarguet, Montferrier-sur-Lez, collections INRA, IRD, CIRAD, SupAgro). Un dépôt à Antananarivo est à l'étude.

Pour l'interprétation des différentes pièces génitales et leur désignation, nous avons utilisé la terminologie de Ross (1956), à l'exception des cerci souvent traduit en français par "appendices préanaux ", auquel nous avons préféré « appendices supérieurs » qui correspond mieux à l'usage actuel "superior appendages" (Schmid 1998 ; Munoz-Quesada \& Holzenthal 2008).

Le profil écologique a été établi pour le genre, car le nombre de captures de la plupart des espèces est trop faible pour offrir des conclusions statistiquement valides. Les sites où le genre a été noté sont classés en fonction de l'altitude, de la distance à la source et du versant (Fig. 11). Cette analyse a été décrite et discutée par Randriamasimanana \& Gibon (2001).

\section{ABRÉVIATIONS}

CBGP Centre de Biologie pour la Gestion des Populations, France Campus International de Baillarguet, Montferrier-sur-Lez ;

MNHN Muséum national d'Histoire naturelle, Paris ; LRSAE Laboratoire de Recherches sur les Systèmes Aquatiques et leur Environnement, Antananarivo.

\section{SYSTÉMATIQUE}

Famille Philopotamidae Stephens, 1829 Sous-famille RossODINAE

Özdikmen \& Darilmaz, 2008

Genre Rossodes Öezdikmen \& Darilmaz, 2008

Paulianodes Ross, 1956: 54, figs 19, 22 a-e. — Gibon \& Elouard 1996: 508, 509, 510, 512, 516. — Gibon et al. 


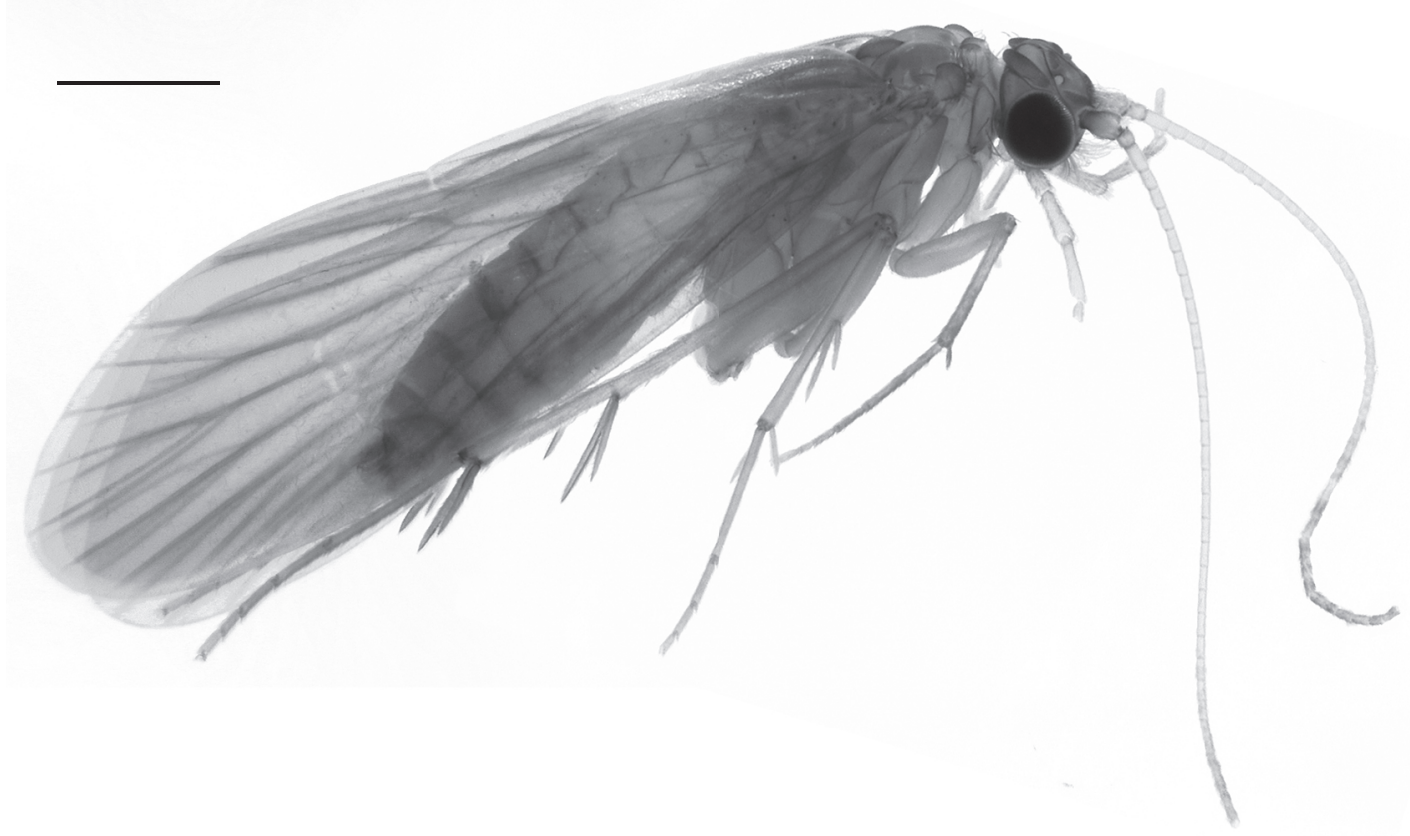

FIG. 1. - Rossodes hertui, mâle, rivière des makis, 2.IV.1994. Photographie: H.P. Aberlenc (C.B.G.P.). Échelle : 1 mm.

1996: 116-119; 1999: 123, 124. — Gibon \& Andriambelo 1999: 98, 99, 101. — Gibon 2000:329. — Blahnik 2002: 124. - Kjer et al. 2002: 86, 88. — Blahnik 2005: 5, 6. - Holzenthal et al. 2007: 665.

non Paulianodes Mamet, 1954:1-86.

ESPÈCE TYPE. - Paulianodes tsaratananae Ross, 1956 par désignation originale.

\section{Distribution. - Genre endémique de Madagascar.}

DiaGNOSE. - Habitus (Fig. 1). Couleur générale brune, ailes uniformément brunes. Tête : trois ocelles, deux grandes verrues postérieures. Palpes labiaux de trois segments, le troisième annelé et plus long que les deux premiers. Palpes maxillaires de cinq segments. Cinquième segment annelé et un peu plus long que les autres, longueur égale à celle des troisième et quatrième réunis. Formule calcarienne : 2/4/4. Aile antérieure : furcas $1,2,3,4$ et 5 présentes ; extrémité de la $\mathrm{Cu} 2$ courbée postérieurement de façon à ce que le point de jonction au bord de l'aile soit proche de celui de A1; A2 et A3 courbées puis fusionnées avec A1. Aile postérieure : furcas 1, 2, 3 et 5 toujours présentes ; la 4 est parfois présente mais généralement absente ; trois veines anales atteignant le bord de l'aile. Genitalia mâles : septième sternite muni d'une petite projection ventro- distale. Neuvième segment abdominal variable, présentant, suivant les espèces, plusieurs stades d'évolution depuis une forme en anneau (neuvième tergite distinct du dixième), jusqu'à la disparition complète du neuvième tergite (parfois transformé en bande membraneuse ou fusionné avec le dixième). Réduction du neuvième tergite accompagnée par l'allongement des zones antéro-latérales du sternite. Appendices inférieurs fortement modifiés : les deux articles 1 (le droit et le gauche) fusionnés sur toute leur longueur, bord disto-ventral de l'ensemble ainsi formé portant une zone dense de petits spicules épais, articles 2 (harpago) présents et indépendants, de taille réduite, leur extrémité portant une petite zone de spicules. Appendices supérieurs de forme variable insérés sur la base du dixième tergite ou fusionnés avec le prolongement distal des extrémités antéro-ventrales du dixième tergite. Bloc central du dixième tergite massif, en toit au-dessus de l'appareil phallique. Appareil phallique tubulaire à base élargie, muni d'un nombre variable d'épines internes et, parfois, d'un sclérite phallotrémal.

\section{Rossodes mantadia n. sp.}

(Fig. 2A-D)

Matériel TYPe. - Holotype : Parc national Mantadia, 14.IV.1995, F.-M. Gibon, 1 ơ (MNHN). 

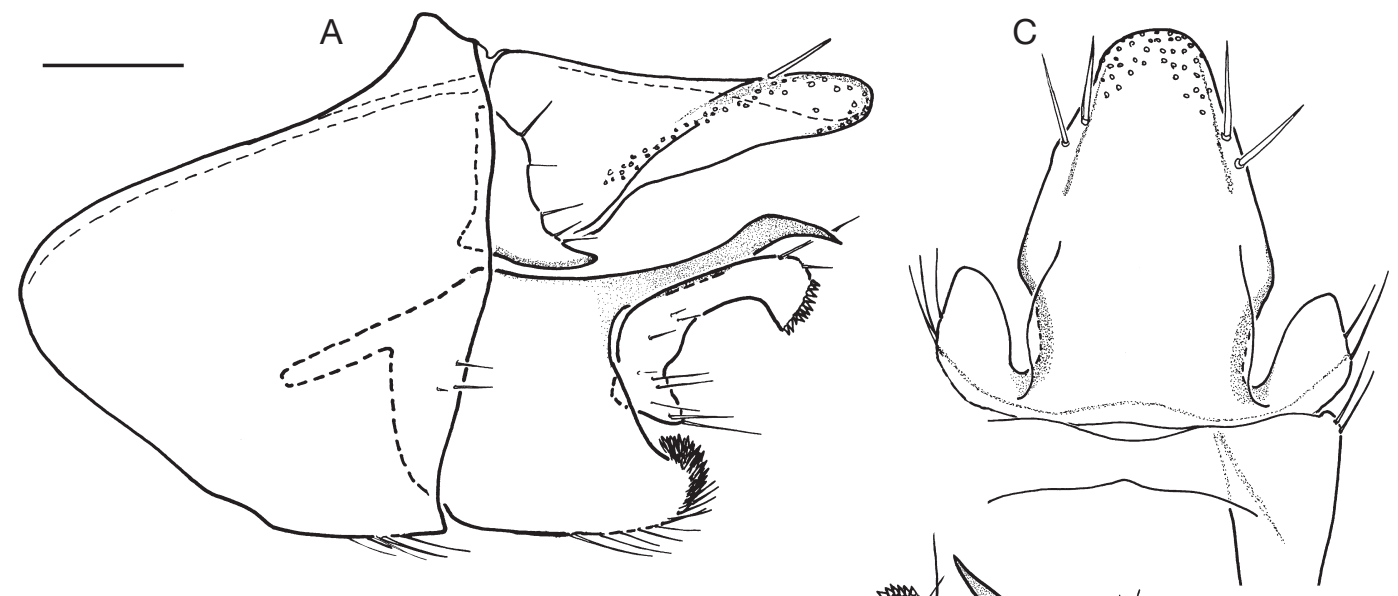

$\mathrm{B}$

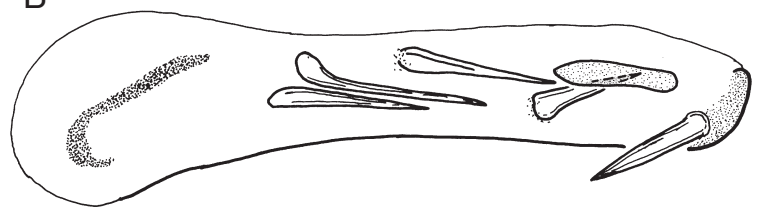

$\mathrm{D}$
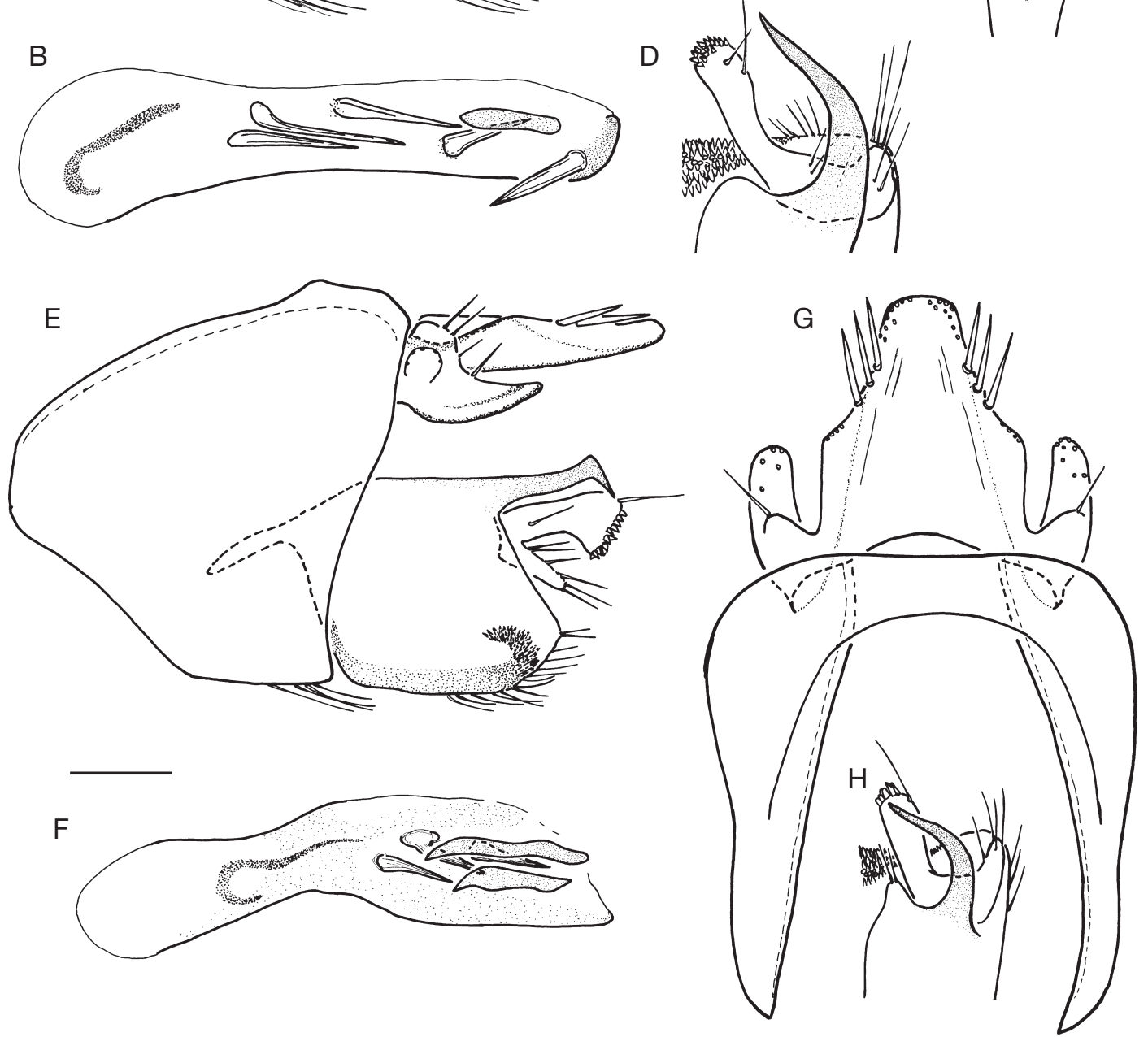

FIG. 2. - A-D, Rossodes mantadia n. sp.; A, vue latérale des genitalia ; B, vue latérale de l'appareil phallique ; C, vue dorsale du dixième tergite ; D, vue dorsale partielle des appendices inférieurs; E-H, R. pilakai n. sp. ; E, vue latérale des genitalia; $\mathbf{F}$, vue latérale de l'appareil phallique ; G, vue dorsale du dixième tergite et du neuvième segment ; H, vue dorsale partielle des appendices inférieurs. Échelles : 0,1 mm. 
LOCALITÉ TYPE. - Madagascar, quelques kilomètres au nord d'Andasibe, sur la Sahatany (Bassin du Rianila), $48^{\circ} 25^{\prime} 57^{\prime \prime} \mathrm{E}, 18^{\circ} 49^{\prime} 41^{\prime \prime} \mathrm{S}$.

DisTRIBUTION. - La distribution connue se limite à la localité type (Fig. 10).

ÉTYMOLOGIE. - Cette espèce est nommée en référence au Parc national Mantadia.

Diagnose. - Rossodes mantadia n. sp. constitue, avec, $R$. pilakai n. sp., $R$. goodmani n. sp., et $R$. hertui n. sp., un groupe d'espèces très proches les unes des autres. Ce sont les seules, avec $R$. tsaratananae, à présenter un neuvième tergite distinct et indépendant du dixième. Chez $R$. mantadia n. sp. et $R$. pilakai n. sp., l'extrémité des appendices supérieurs est arrondie en vue dorsale, alors qu'elle est pointue chez $R$. goodmani n. sp. et $R$. hertui n. sp. $R$. mantadia n. sp. se distingue de $R$. pilakai n. sp. par la forme du lobe médian du dixième tergite abdominal (vue dorsale); ce dernier est en forme de cône à l'extrémité arrondie (Fig. 2C) alors que, chez $R$. pilakai n. sp., il présente, vers la mi-longueur, un rétrécissement accentué (Fig. 2G). On notera également la présence de six paires d'épines internes à l'appareil phallique alors qu'elles sont cinq chez $R$. pilakai n. sp.

\section{DESCRIPTION}

Taille. Aile antérieure : $0,57 \mathrm{~cm}$, aile postérieure : $0,51 \mathrm{~cm}$.

Genitalia mâles. Bord antérieur latéral du neuvième segment abdominal déformé, allongé et profondément invaginé dans le huitième; ce qui donne à ce segment une forme grossièrement triangulaire en vue latérale. Neuvième tergite sclérifié et distinct du dixième, réduit à une bande dorsale en forme d'anse. Appendices inférieurs : structure caractéristique du genre : les deux articles 1 fusionnés en un bloc massif portant latéro-distalement l'article 2 ; zone dense de spicules fortement sclérifiées sur la partie médiane du bord ventro-distal ; bords dorsaux déformés, prolongés en un lobe spiniforme distal courbé vers l'intérieur. Appendices supérieurs : dorsalement en forme de courtes projections latéro-distales ovoïdes insérées sur la base du dixième tergite, latéralement, de forme triangulaire avec une extrémité distale spiniforme. Dixième tergite : élément central massif en toit au-dessus de l'appareil phallique ; extrémité distale arrondie ; nombreuses sensilles distales, ligne latérale de sensilles, deux soies plus longues vers l'extrémité de chaque ligne latérale. Appareil phallique : six paires d'épines internes et un sclérite phallotrémal.

\section{Rossodes pilakai n. sp.}

(Fig. 2E-H)

Matériel type. - Holotype : Madagascar, Fenoevo, 15.IV.1992, J.-M. Elouard, 1 ơ (MNHN). Paratype : Imonty sur l'Imonty (Bassin du Mandrare), 4643'18"E, 2449'03"S, 22.V.1994, F.-M. Gibon, $10^{\circ}$ (CBGP). - Réserve d'Andohahela, sur l'Ambahibe (Bassin de la Efaho), 4651'09”E, 2445’07”S, 17.V.1994, F.-M. Gibon, 1 ơ (CBGP).

Autre matériel examiné. - Camp 2 du WWF, dans la réserve d'Andohahela, sur l'Andranohela (Bassin de

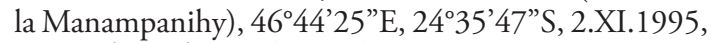
J.-M. Elouard, $10^{\circ}$ (CBGP).

LOCALITÉ TYPE. - Madagascar, près de Fenoevo, sur un tributaire de la Manampanihy, 46053'39”E, 24'41'00”S.

DisTRibution. - La distribution connue se limite au massif d'Andohahela, au sud-est de Madagascar (Fig. 10).

ÉTYMOLOGIE. — Cette espèce est dédiée à Théogène Pilaka, qui travaillait au LRSAE sur les Simuliidae malgaches.

Diagnose. - Rossodes pilakai n. sp. et $R$. mantadia n. sp. sont les seules espèces à présenter à la fois un neuvième tergite distinct et indépendant du dixième et l'extrémité des appendices supérieurs arrondie en vue dorsale. Rossodes pilakai n. sp. se distingue par la présence d'un rétrécissement accentué de l'élément central du dixième tergite (Fig. 2G). Elle est également caractérisée par l'élargissement de la partie distale de l'appareil phallique (Fig. 2F). On notera, de plus, la présence de cinq paires d'épines internes à l'appareil phallique alors qu'elles sont six chez $R$. mantadia n. sp.

\section{DESCRIPTION}

Taille. Aile antérieure : $0,56 \mathrm{~cm}$, aile postérieure : de 0,49 à $0,50 \mathrm{~cm}$.

Genitalia mâles. Bord proximal du neuvième segment abdominal arrondi, convexe, profondément invaginé dans le huitième. Neuvième tergite réduit mais présent, sclérifié et distinct du dixième. Appendices inférieurs : structure caractéristique du genre, bords dorso-externes déformés en un lobe spiniforme courbé vers l'intérieur. Appendices supérieurs, apparaissant sur la vue dorsale comme de courtes projections latéro-distales digitiformes 
de la base du dixième tergite, munis d'une petite excroissance latérale bulbeuse ; en vue latérale : forme grossièrement triangulaire, extrémité distale spiniforme. Dixième tergite composé de deux petits éléments latéraux (fusionnés avec les appendices supérieurs) et d'un grand élément central massif à l'extrémité distale arrondie ; il porte de nombreuses sensilles distales, une ligne latérale de sensillae ainsi que trois soies. Appareil phallique muni d'un sclérite phallotrémal et de cinq paires d'épines internes, deux d'entre elles légèrement plus allongées et nettement élargies en forme de bâtonnet.

\section{Rossodes hertui n. sp.}

(Fig. 3A-D)

Matériel type. - Holotype : en aval du Camp base WWF, Parc national de la Montagne d'Ambre, 31.III.1995, J.-M. Elouard, 1 ơ (MNHN).

Paratype : mêmes localité et date que l'holotype, J.-M. Elouard, 1 o' (CBGP). — Aval du Camp base WWF, Parc national de la Montagne d'Ambre, rivière des Makis (Bassin de l'Antongombato), 49 $10^{\prime} 21^{\prime \prime E} 12^{\circ} 31^{\prime} 38^{\prime \prime} S$, 29.III.1994, J.-M. Elouard, $10^{7}$ (CBGP). - Matsabory-Malio (Bassin de l'Antongombato), 4909'36"E, $12^{\circ} 35^{\prime} 45^{\prime \prime}$ S, 03.V.1995, J.-M. Elouard, 2 o" o" (CBGP).

Autre MATÉRIEL EXAMiné. - Aval de la grande cascade, rivière des Makis (Bassin de l'Antongombato), 49 $10^{\circ} 14$ 'E 12²9’17”S, 2.IV.1994, 1 ơ (CBGP).

LOCALITÉ TYPE. - Madagascar, Montagne d'Ambre, rivière des Makis (Bassin de l'Antongombato), 49 $10^{\circ} 21^{\prime \prime} \mathrm{E}$ $12^{\circ} 31^{\prime} 38^{\prime \prime}$ S.

DisTRIBUTION. - La distribution connue se limite à la Montagne d'Ambre (Fig. 10).

ÉTYMOLOGIE. - Cette espèce est dédiée à Olivier Hertu, informaticien au LRSAE.

DiagnOSE. - Rossodes hertui n. sp. et $R$. goodmani n. sp. sont les seules espèces à présenter à la fois un neuvième tergite distinct et indépendant du dixième et l'extrémité des appendices supérieurs pointue (vue dorsale). Elles se distinguent par la forme de l'extrémité du dixième tergite, arrondie chez $R$. hertui n. sp. (Fig. 3C), bifide chez R. goodmani n. sp. (Fig. 3G).

\section{DESCRIPTION.}

Taille. Aile antérieure : de 0,61 à $0,63 \mathrm{~cm}$, aile postérieure : $0,55 \mathrm{~cm}$.
Genitalia mâles. Bord antérieur du neuvième segment abdominal arrondi, convexe et profondément invaginé dans le huitième. Neuvième tergite réduit mais sclérifié et distinct du dixième. Appendices inférieurs : structure caractéristique du genre, bords dorso-externes déformés en un lobe spiniforme courbé vers l'intérieur. Appendices supérieurs, apparaissant dorsalement comme des projections latéro-distales spiniformes de la base du dixième tergite, munis d'une excroissance latérale bulbeuse ; longueur légèrement supérieure à la moitié de celle du dixième tergite; en vue latérale : forme de poignard courbé dorsalement. Dixième tergite composé de deux petits éléments latéraux (fusionnés avec les appendices supérieurs) et d'un grand élément central massif à l'extrémité distale arrondie, il porte de nombreuses sensilles distales, une ligne latérale de sensilles et trois soies. Appareil phallique muni de cinq paires d'épines internes, trois d'entre elles plus fines et plus allongées.

\section{Rossodes goodmani n. sp.}

(Fig. 3E-H)

MATÉRIEL TYPE. - Holotype : Camp 2 du WWF, réserve nationale de l'Andringitra, 21.XI.1993, F-M. Gibon, 1 o" (MNHN).

Paratype : Camp 3 du WWF, réserve nationale de l'Andringitra, sur un affluent de la Sahavatoy (Bassin de

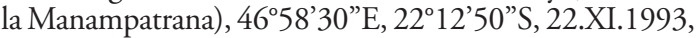
F-M. Gibon, 1 o" (CBGP).

LOCALITÉ TYPE. - Madagascar, massif de l'Andringitra, sur un petit tributaire de la Sahavatoy (Bassin de la

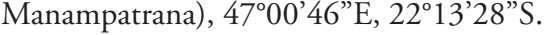

DisTRIBUTION. - La distribution connue se limite au massif de l'Andringitra (Fig. 10).

ÉTYMOLOGIE. - Cette espèce est dédiée à Steve Goodman, en témoignage de notre reconnaissance.

Diagnose. - Voir la diagnose de Rossodes hertui n. sp.

\section{DESCRIPTION}

Taille. Aile antérieure : $0,54 \mathrm{~cm}$, aile postérieure : $0,48 \mathrm{~cm}$.

Genitalia mâles. Bord proximal du neuvième segment abdominal très déformé latéralement et profondément invaginé dans le huitième. Neuvième 
tergite réduit mais sclérifié et distinct du dixième. Appendices inférieurs : structure caractéristique du genre, bords dorso-externes déformés en un lobe spiniforme courbé vers l'intérieur. Appendices supérieurs, vue dorsale : projections latéro-distales spiniformes de la base du dixième tergite ; longueur légèrement supérieure à la moitié de celle du dixième tergite; vue latérale : forme de gros poignard courbé dorsalement. Dixième tergite composé de deux petits éléments latéraux (fusionnés avec les appendices supérieurs) et d'un grand élément central massif dont l'extrémité distale arrondie offre une invagination en $\mathrm{U}$; nombreuses sensilles distales, de chaque côté : une ligne latérale de sensilles terminée par trois soies. Appareil phallique : un sclérite phallotrémal et cinq paires d'épines internes, l'une d'entre elles légèrement élargie.

\section{Rossodes fabienneae n. sp.}

(Fig. 4A-D)

MATÉRIEL TYPE. - Holotype : Camp 3 du WWF, réserve nationale de l'Andringitra, F.-M. Gibon, $10^{\prime \prime}$ (MNHN).

LOCALITÉ TYPE. - Madagascar, massif de l'Andringitra, sur un affluent de la Sahavatoy (Bassin de la Manampatrana), $46^{\circ} 58^{\prime} 30^{\prime \prime} \mathrm{E}, 22^{\circ} 12^{\prime} 50^{\prime \prime} \mathrm{S}$.

DisTRIBUTION. - La distribution connue se limite au massif de l'Andringitra (Fig. 10).

ÉTYMologiE. - Cette espèce est dédiée à Fabienne Ranaivoharindraka, l'une des pionnières de l'étude des Trichoptères au LRSAE.

DiagnOSE. - Rossodes fabienneae n. sp. est proche de $R$. manantenina $\mathrm{n}$. sp. ; elle s'en distingue par la longueur des appendices supérieurs, plus des trois-quarts de celle du dixième tergite, alors qu'elle n'est égale qu'à la moitié chez $R$. manantenina $\mathrm{n}$. sp. On note également la présence de deux paires d'épines internes à l'appareil phallique et de six chez $R$. manantenina $\mathrm{n}$. sp.

\section{DESCRIPTION}

Taille. Aile antérieure : $0,68 \mathrm{~cm}$, aile postérieure : $0,62 \mathrm{~cm}$.

Genitalia mâles. Latéralement, bord antérieur du neuvième segment abdominal arrondi et profondément invaginé dans le huitième. Neuvième tergite réduit à une mince bande peu sclérifiée et difficilement distincte du bord antérieur du dixième tergite. Appendices inférieurs : structure caractéristique du genre, bords dorso-externes déformés en un lobe spiniforme en forme de serpe. Appendices supérieurs insérés à la base du dixième tergite, sclérifiés, allongés distalement, en forme de sabre (vue latérale) ; longueur égale aux trois quarts de celle du dixième tergite. Dixième tergite composé de deux petits éléments latéraux (fusionnés avec les appendices supérieurs) et d'un grand élément central massif à l'extrémité distale arrondie, nombreuses sensilles distales, de chaque côté un groupe de trois soies alignées. Appareil phallique muni d'un mince sclérite phallotrémal et de deux paires de courtes épines internes.

\section{Rossodes manantenina $\mathrm{n}$. sp.}

(Fig. 4E-H)

Matériel tyPe. - Holotype : Camp 2 du WWF, Parc national du Marojejy, 17.X.1996, J. Legrand, D. Randriamasimanana, $10^{\top}$ (MNHN).

Paratypes : Mêmes localité et date que l'holotype, J. Legrand, D. Randriamasimanana, 1 o (CBGP). Même localité que l'holotype, 22.X.1996, J. Legrand, D. Randriamasimanana, 1 ơ (CBGP).

Autre matériel examiné. - Camp 1 du WWF, Parc national du Marojejy, sur un affluent de la Manantenina (Bassin de la Lokoho), 4946'20”E, 1426’02”S, 13.X.1996, 1 ơ (CBGP).

LOCALITÉ TYPE. - Madagsacar, massif du Marojejy, sur un affluent de la Manantenina (Bassin de la Lokoho), $49^{\circ} 45^{\prime} 37^{\prime \prime} \mathrm{E}, 14{ }^{\prime} 25^{\prime} 57^{\prime \prime} \mathrm{S}$.

Distribution. - La distribution connue se limite au massif du Marojejy.

ÉTYMOLOGIE. - Cette espèce est nommée en référence à la rivière Manantenina.

DiAgnOSE. - Voir la diagnose de Rossodes fabienneae n. sp.

\section{DESCRIPTION}

Taille. Aile antérieure : $0,58 \mathrm{~cm}$, aile postérieure : $0,52 \mathrm{~cm}$.

Genitalia mâles. Latéralement, bord antérieur du neuvième segment abdominal arrondi et invaginé dans le huitième. Neuvième tergite réduit, peu sclérifié, distinct du dixième, ouvert dorsalement. 

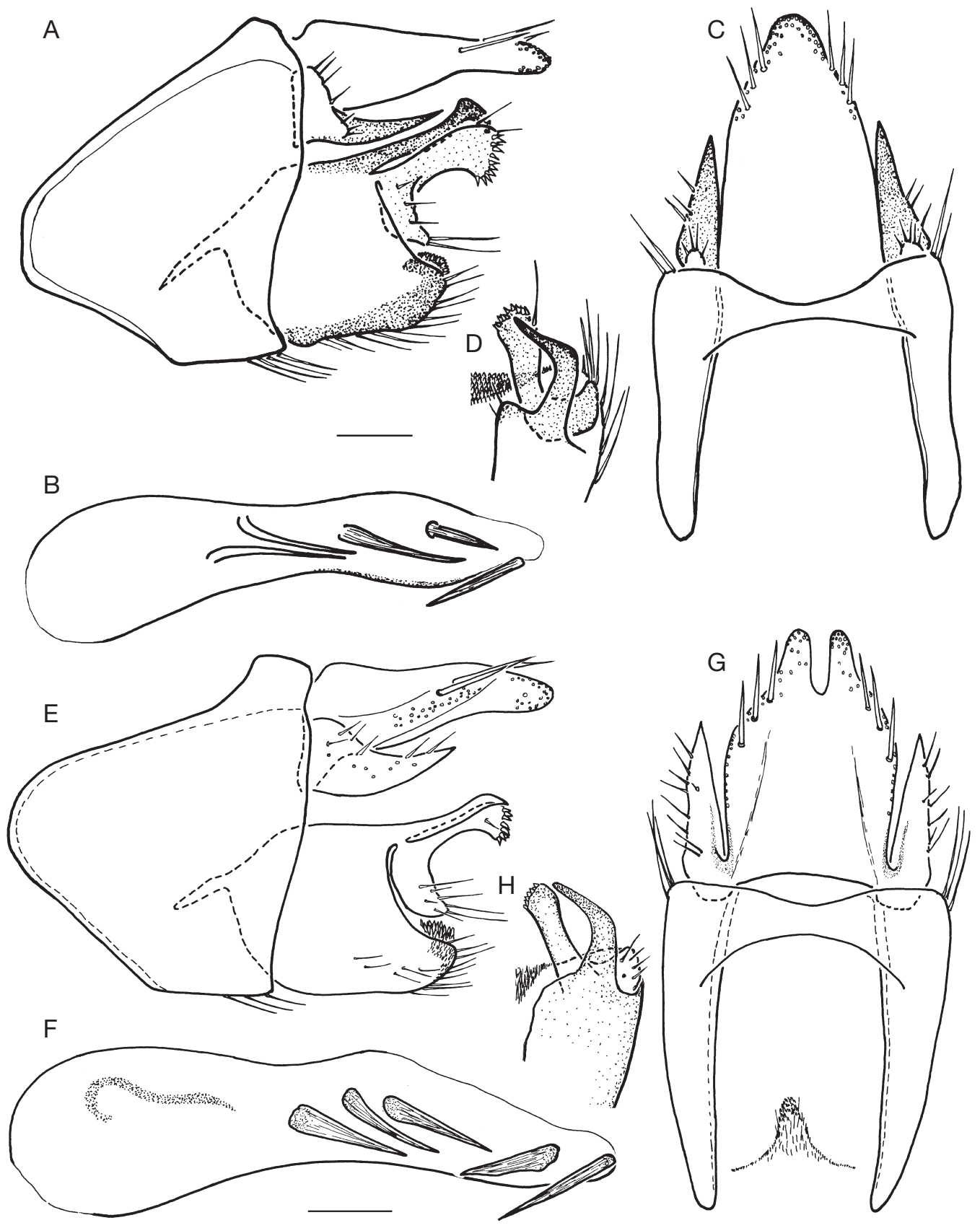

FIG. 3. - A-D, Rossodes hertui n. sp. : A, vue latérale des genitalia; B, vue latérale de l'appareil phallique ; C, vue dorsale du dixième tergite; $\mathbf{D}$, vue dorsale partielle des appendices inférieurs. E-H, R. goodmani n. sp. ; E, vue latérale des genitalia; $\mathbf{F}$, vue latérale de l'appareil phallique ; G, vue dorsale du dixième tergite et du neuvième segment ; $\mathbf{H}$, vue dorsale partielle des appendices inférieurs. Échelles : 0,1 mm. 
Appendices inférieurs : structure caractéristique du genre, bords dorso-externes déformés en un lobe spiniforme sinueux (vue dorsale). Appendices supérieurs insérés à la base du dixième tergite, en forme de poignard courbé dorsalement (vue latérale), munis d'une petite zone bulbeuse latérale portant quelques courtes soies; longueur légèrement supérieure à la moitié de celle du dixième tergite. Dixième tergite composé de deux petits éléments latéraux (fusionnés avec les appendices supérieurs) et d'un grand élément central massif à l'extrémité distale arrondie, un groupe de sensillae distales, une ligne latérale de trois soies (de chaque côté). Appareil phallique muni d'un sclérite phallotrémal et de six paires d'épines internes, l'une d'entre elles de forme atypique, plus courte et plus large en vue latérale.

\section{Rossodes andohahela n. sp.}

(Fig. 5A-D)

MATÉRIEL TYPE. - Holotype : Camp 1 du WWF, réserve nationale d'Andohahela, 25.XI.1995, J.-M. Elouard, $10^{\top}(\mathrm{MNHN})$.

Paratype: Réserve nationale d'Andohahela, sur l'Ambahibe (Bassin de la Efaho, 4651'09"'E, 244'07”S), 17.V.1994, F.-M. Gibon, F. Ranaivoharindriaka, $10^{7}$ (CBGP). 46 51'07”E, 2446'17”S, 18.05.1994, F.-M. Gibon, F. Ranaivoharindriaka, $10^{7}$ (CBGP).

Autre MATÉRIEL EXAMiné. - Même localité et date que l'holotype, J.-M. Elouard, 1 ơ (CBGP).

LOCALITÉ TYPE. - Madagascar, massif d'Andohahela, sur la rivière Andranohela (Bassin de la Manampanihy), $46^{\circ} 45^{\prime} 34^{\prime \prime E}, 24^{\circ} 36^{\prime} 43^{\prime \prime}$ S.

Distribution. - Madagascar, massif d'Andohahela.

ÉTYMOLOgie. — Massif d'Andohahela.

DiagnOSE. — La présence d'une projection latérale bifide de part et d'autre du lobe médian du dixième tergite empêche toute confusion avec une autre espèce. La disymétrie entre les deux paires d'épines internes à l'appareil phallique est une autre caractéristique remarquable de cette espèce.

\section{DESCRIPTION}

Taille. Aile antérieure : $0,55 \mathrm{~cm}$, aile postérieure : $0,48 \mathrm{~cm}$.
Genitalia mâles. Bord antérieur du neuvième sternite arrondi, convexe, invaginé dans le huitième. Neuvième tergite fusionné avec la base du dixième. Appendices inférieurs : structure caractéristique du genre, extrémité distale du bord dorso-latéral grossièrement arrondie. Appendices supérieurs insérés à la base du dixième tergite, allongés distalement (longueur à peine inférieure à celle du dixième tergite), élargis à l'extrémité en forme de massue, un ensemble de soies épaisses sur la partie distale. Dixième tergite grossièrement triangulaire (vue dorsale); au-dessus de chaque appendice supérieur : une projection latérale bifide et spiniforme; un groupe de sensillae distales. Appareil phallique muni d'un sclérite phallotrémal, d'une petite zone de spicules et deux paires d'épines internes, l'une (distale) de taille plus réduite que l'autre (moins du quart de la longueur).

\section{Rossodes ambreensis n. sp.}

(Fig. 5E-H)

Matériel tYPe. - Holotype : Camp de base du WWF, Parc national de la Montagne d'Ambre, 3.IV.1995, J.-M. Elouard, 1 ơ (MNHN).

Paratypes : Même localité et date que l'holotype, 28.III.1994, J.-M. Elouard, 6 ơ ơ (CBGP).

AUTRE MATÉRIEL EXAMINÉ. - Une centaine de mètres en aval de la localité type, $49^{\circ} 10^{\prime} 21^{\prime \prime E}, 12^{\circ} 31^{\prime} 38^{\prime \prime}$, 2.IV.1994, J.-M. Elouard, $10^{7}$ (CBGP). — En aval de la grande cascade, rivière des Makis (Bassin de l'Antongombato), 4910'14”E, $12^{\circ} 29^{\prime} 17^{\prime}$ 'S, 2.IV.1994, J.-M. Elouard, $10^{\prime \prime}$ (CBGP).

LOCALITÉ TYPE. - Madagascar, massif de la Montagne d'Ambre, rivière des Makis (Bassin de l'Antongombato), $49^{\circ} 10^{\prime} 09^{\prime \prime} \mathrm{E}, 12^{\circ} 31^{\prime} 40^{\prime \prime} \mathrm{S}$.

Distribution. - La distribution connue se limite au massif de la Montagne d'Ambre.

ÉTYMOLOGIE. - Cette espèce est nommée en référence au massif de la montagne d'Ambre.

DiAGNOSE. — La forme du dixième tergite empêche toute confusion avec une autre espèce. Les deux expensions latérales du lobe médian sont caractéristiques; elles sont épaisses en vue latérale et digitiformes en vue dorsale, très différentes des déformations homologues présentes chez $R$. andohahela n. sp., qui sont minces, bifides et spiniformes. 

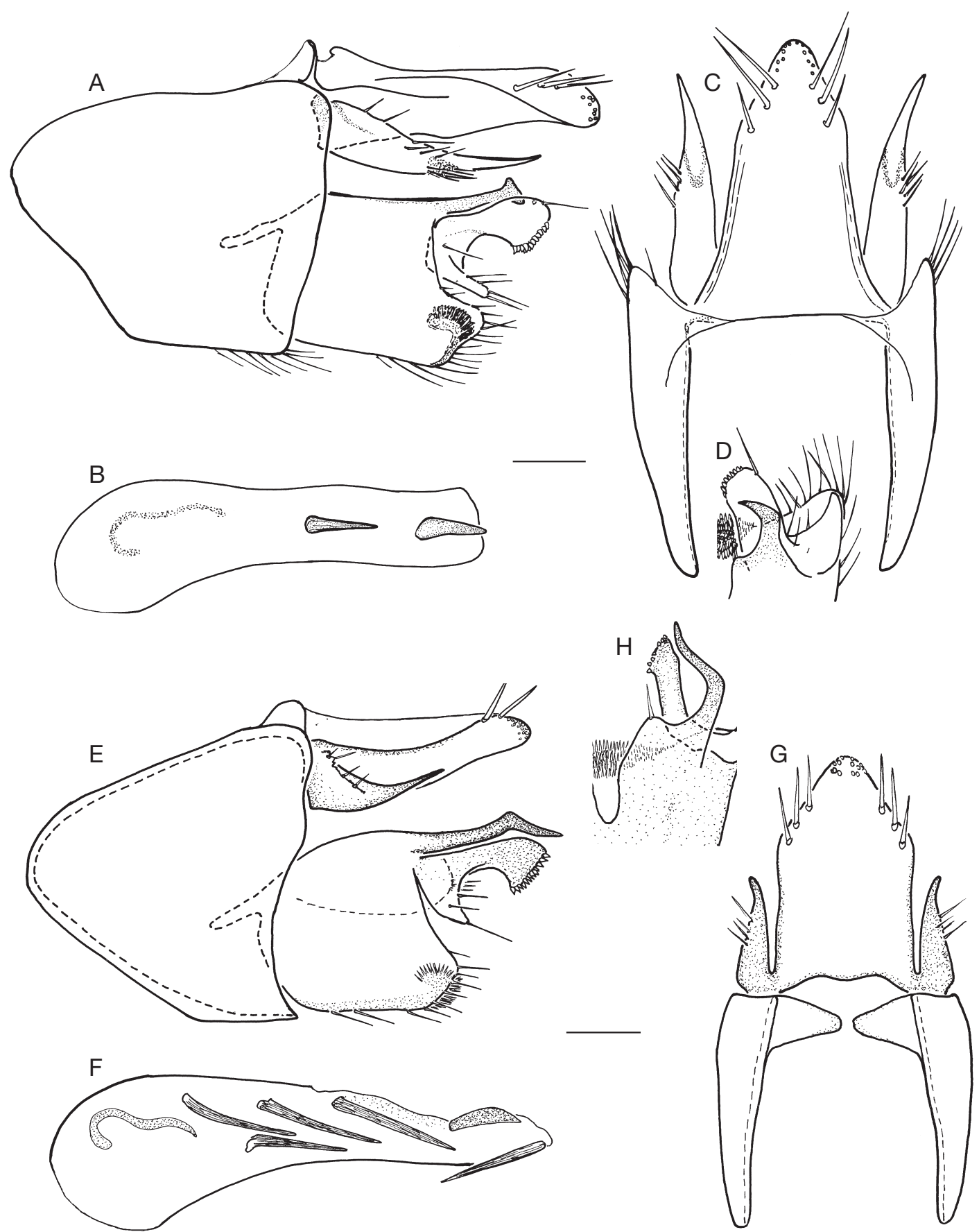

FIG. 4. - A-D, Rossodes fabienneae n. sp. ; A, vue latérale des genitalia ; B, vue latérale de l'appareil phallique ; C, vue dorsale du dixième tergite ; $\mathbf{D}$, vue dorsale partielle des appendices inférieurs ; E-H, R. manantenina $\mathrm{n}$. sp. ; $\mathbf{E}$, vue latérale des genitalia ; $\mathbf{F}$, vue latérale de l'appareil phallique; G, vue dorsale du dixième tergite et du neuvième segment; $\mathbf{H}$, vue dorsale partielle des appendices inférieurs. Échelles : 0,1 mm. 


\section{DESCRIPTION}

Taille. Aile antérieure : de 0,60 à $0,61 \mathrm{~cm}$, aile postérieure : de 0,52 à $0,55 \mathrm{~cm}$.

Genitalia mâles. Bord antérieur du neuvième sternite déformé et invaginé dans le huitième, en forme de triangle tronqué (vue latérale). Neuvième tergite fusionné avec la base du dixième. Appendices inférieurs : structure caractéristique du genre, extrémité distale du bord dorso-latéral grossièrement arrondie. Appendices supérieurs insérés à la base du dixième tergite, allongés distalement (longueur à peine inférieure à celle du dixième tergite), élargis à l'extrémité en forme de massue, un ensemble de soies épaisses sur la partie distale. Dixième tergite massif, forme grossièrement triangulaire (vue dorsale), une carène centrale saillante qui se divise en deux gros lobes latéraux terminés par une courte soie. Appareil phallique large et massif (vue latérale), un sclérite phallotrémal et quatre paires d'épines internes effilées et légèrement courbées.

\section{Rossodes namorona n. sp.}

(Fig. 6A-D)

MATÉRIEL TYPE. - Holotype : Ranomafana, 6.XI.1996, F.-M. Gibon, 1 ơ (MNHN).

AUtRe MATÉRIEL EXAMinÉ. - Ranomafana, sur un affluent de Namorona, 47 25'23"E, $21^{\circ} 15^{\prime} 43^{\prime \prime} S$, 6.11.1996, F.-M. Gibon, $10^{\circ}$ (CBGP). — Zone de recherche du Parc national, affluent de la Namorona, $47^{\circ} 25^{\prime} 15^{\prime \prime}$ E $21^{\circ} 15^{\prime} 50^{\prime}$ 'S 7.XI.1996, F.-M. Gibon, $10^{\prime}(\mathrm{CBGP})$.

LOCALITÉ TYPE. - Ranomafana, sur un petit affluent

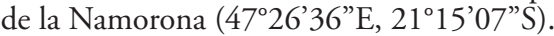

Distribution. - Madagascar, haute vallée de la Namorona.

ÉTYMOLOGIE. - Cette espèce est nommée en référence au fleuve Namorona.

DIAGNOSE. - Rossodes namorona $\mathrm{n}$. sp. et $R$. marojejyensis n. sp. sont les seules espèces à présenter à la fois des appendices supérieurs allongés digitiformes et des lobes latéraux du dixième segment allongés spiniformes. Elles se distinguent entre elles par la forme de l'extrémité distale du dixième tergite (un étranglement chez $R$. namorona n. sp., deux chez $R$. marojejyensis n. sp.), ainsi que par le nombre de paires d'épines internes à l'appareil phallique (quatre chez $R$. namorona $\mathrm{n}$. sp., trois chez $R$. marojejyensis n. sp.)

\section{DESCRIPTION}

Taille. Aile antérieure : $0,46 \mathrm{~cm}$, aile postérieure : $0,41 \mathrm{~cm}$.

Genitalia mâles. Bord antérieur du neuvième sternite arrondi et invaginé dans le huitième. Neuvième tergite fusionné avec la base du dixième. Appendices inférieurs : structure caractéristique du genre; second article épais, en forme de $\mathrm{V}$ aux branches inégales (vue dorsale). Appendices supérieurs insérés à la base du dixième tergite, en forme de long lobe digitiforme (longueur à peine inférieure à celle du dixième tergite), un groupe de soies distales. Extrémités antérieures du dixième tergite déformées en un long lobe spiniforme, mince, dirigé distalement puis courbé dorsalement (vue latérale) et vers l'extérieur (vue dorsale). Élément central massif, progressivement aminci, présentant, peu avant l'extrémité distale, un étranglement qui donne à l'extrémité une forme caractéristique "en tétine ». Appareil phallique : un sclérite phallotrémal, deux petites zones de spicules, quatre paires d'épines droites et plutôt fines, une épine isolée, probablement surnuméraire.

\section{Rossodes marojejyensis $\mathrm{n}$. sp.}

(Fig. 6E-H)

Matériel type. - Holotype : Camp 3 du WWF, Parc national du Marojejy, 27.X.1996, J. Legrand, D. Randriamasimanana, $10^{\prime \prime}$ (MNHN).

Autre matériel examiné. - Camp 4 du WWF, Parc national du Marojejy, sur l'Andranomifotatra (Bassin de la Lokoho), 4944'05”'E, 14²6'04”S, 12.XI.1996, J. Legrand, D. Randriamasimanana, $10^{7}$.

LOCALITÉ TYPE. - Madagascar, massif du Marojejy, sur l'Andranomifotatra (Bassin de la Lokoho), 4944'05'E, $14^{\circ} 26^{\prime} 02^{\prime \prime} \mathrm{S}$.

DistribUtion. - La distribution connue se limite au massif du Marojejy.

ÉTYMOLOGIE. - Cette espèce est nommée en référence au massif du Marojejy. 
A

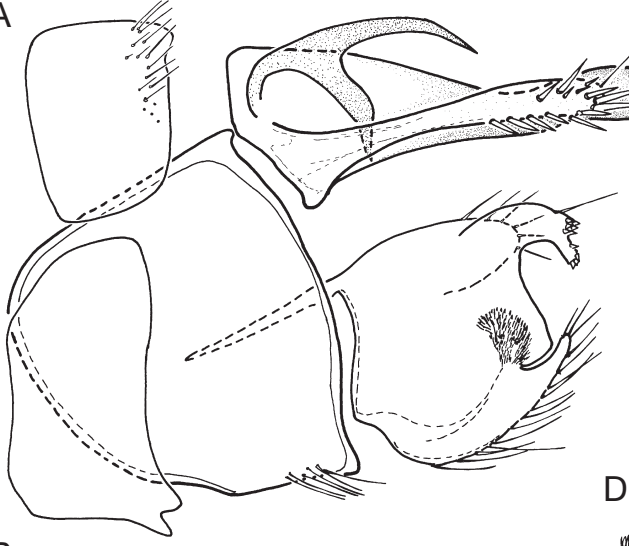

B

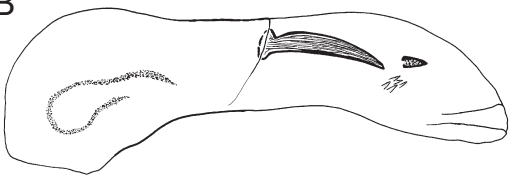

$\mathrm{F}$
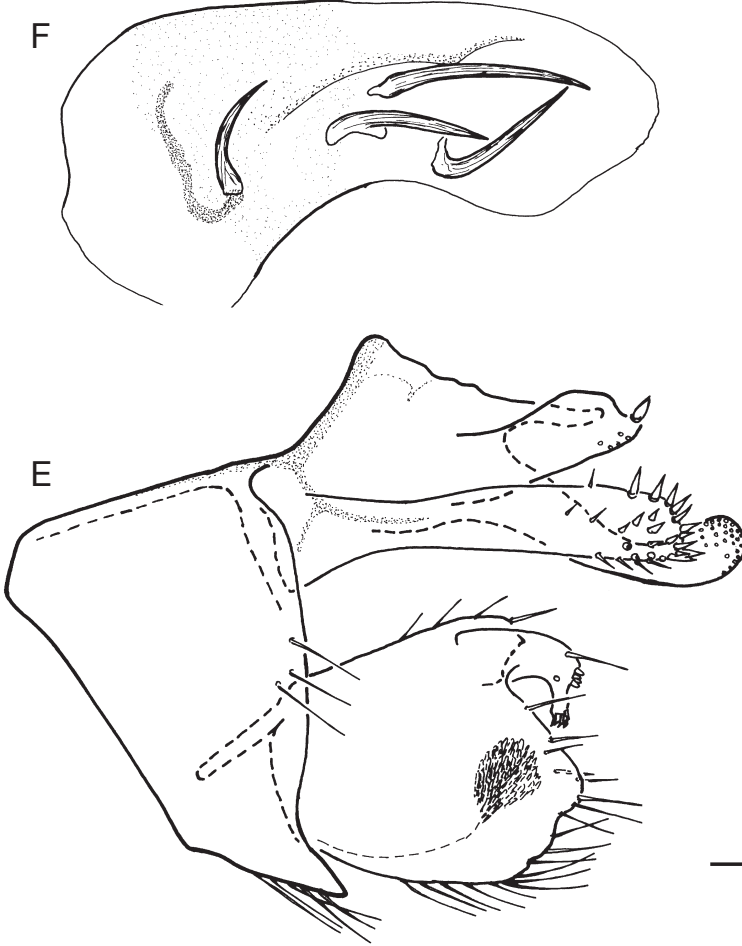

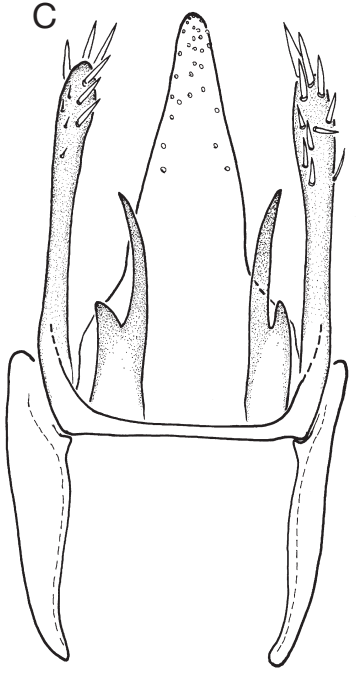

D
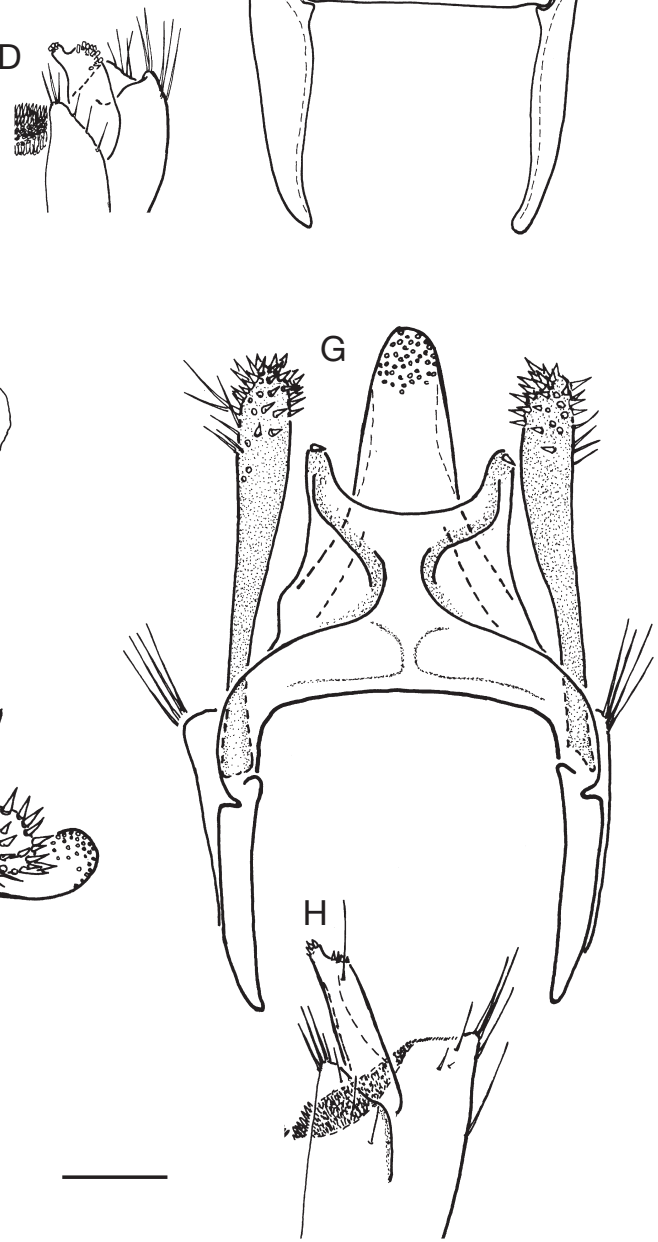

FIG. 5. - A-D, Rossodes andohahela n. sp. : A, vue latérale des genitalia ; B, vue latérale de l'appareil phallique ; C, vue dorsale du dixième tergite ; $\mathbf{D}$, vue dorsale partielle des appendices inférieurs ; E-H, R. ambreensis n. sp. ; E, vue latérale des genitalia ; $\mathbf{F}$, vue latérale de l'appareil phallique; G, vue dorsale du dixième tergite et du neuvième segment; $\mathbf{H}$, vue dorsale partielle des appendices inférieurs. Échelles : 0,1 mm. 
DiAgnOSE. - Rossodes marojejyensis $\mathrm{n}$. sp. est une espèce proche de $R$. namorona n. sp. Elle s'en distingue par la présence de deux étranglements successifs à l'extrémité du lobe médian du dixième tergite (un seul chez $R$. namorona n. sp.), ainsi que par celle de trois paires d'épines internes à l'appareil phallique (quatre chez $R$. namorona n. sp.).

\section{DESCRIPTION}

Taille. Aile antérieure : $0,54 \mathrm{~cm}$, aile postérieure : $0,48 \mathrm{~cm}$.

Genitalia mâles. Neuvième tergite fusionné avec la base du dixième. Appendices inférieurs : structure caractéristique du genre ; second article courbé en forme de $U$ (vue dorsale). Appendices supérieurs insérés à la base du dixième tergite, en forme de long lobe digitiforme (longueur à peine inférieure à celle du dixième tergite), un groupe de soies distales. Extrémités antérieures du dixième tergite déformées en un long lobe spiniforme, mince, dirigé distalement puis courbé dorsalement (vue latérale). Partie centrale caractérisée par une carène dorsale qui se termine par un bec aux trois-quarts de la longueur (vue latérale). Extrémité distale : un groupe de sensillae, deux étranglements successifs (vue dorsale) encadrant un groupe de deux soies. Appareil phallique : sclérite phallotrémal, deux longues zones de spicules et trois paires d'épines internes, base des épines élargie en tête-de-clou.

\section{Rossodes langrandi $\mathrm{n}$. sp.}

(Fig. 7A-D)

MATÉRIEL TYPE. - Holotype : Camp 4 du WWF, réserve nationale de l'Andringitra, 24.XI.1993, F.-M. Gibon, $10^{7}$ (MNHN).

LOCALITÉ TYPE. - Madagascar, massif de l'Andringitra, sur un petit tributaire de la Manampatrana, $46^{\circ} 57^{\prime} 50^{\prime \prime} \mathrm{E}$, $22^{\circ} 12^{\prime} 00^{\prime \prime} S$.

DisTRIBUTION. - La distribution connue se limite au massif de l'Andringitra.

Étymologie. - Cette espèce est dédiée à Olivier Langrand.

DiAGNOSE. - Rossodes langrandi n. sp. est la seule espèce à présenter à la fois des lobes latéraux du dixième segment allongés, spiniformes et des appendices supérieurs courts et en forme de bosse.

\section{DESCRIPTION}

Taille. Aile antérieure : $0,75 \mathrm{~cm}$, aile postérieure : $0,66 \mathrm{~cm}$.

Genitalia mâles. Bords antérieurs du neuvième sternite développés, convexes et invaginés dans le huitième. Neuvième tergite disparu, fusionné avec la base du dixième. Appendices inférieurs : structure caractéristique du genre; second article court épais et courbé en U (vue dorsale). Appendice supérieur réduit à une bosse couverte de soies allongées insérée sur la base du dixième tergite. Extrémités antérieures du dixième tergite déformées en un long lobe spiniforme, mince, dirigé disto-dorsalement (vue latérale). Élément central du dixième tergite grossièrement triangulaire (vue latérale), une courte carène dorsale sur la partie basale, en forme de tétine sur la partie distale (vue dorsale). Appareil phallique : sclérite phallotrémal indistinct, une paire d'épines internes.

\section{Rossodes humberti $\mathrm{n}$. $\mathrm{sp}$.}

(Fig. 7E-H)

Matériel tYPe. - Holotype : Camp 2 du WWF, Parc national du Marojejy, 22.X.1996, J. Legrand, D Randriamasimanana, 1 o" (MNHN).

Paratypes : Camp 3 du WWF, Parc national du Marojejy, sur l'Andranomifotatra (Bassin de la Lokoho), 4944'05”'E, 1426'02"S, 27.X.1996, J. Legrand, D. Randriamasimanana, $20^{\prime} 0^{\prime \prime}$ (CBGP)

LOCALITÉ TYPE. - Madagascar, massif du Marojejy, sur l'Andranomifotatra (Bassin de la Lokoho), 49 45'07'E, $14^{\circ} 26^{\prime} 00^{\prime \prime} S$.

Distribution. - La distribution connue se limite au massif du Marojejy.

ÉTYMOLOGIE. - Cette espèce est dédiée au botaniste Henri Humbert qui organisa la première mission scientifique sur le massif du Marojejy.

DiAgNOSE. - Rossodes humberti n. sp. est une espèce proche de $R$. rakotonirinai $\mathrm{n}$. sp. et de $R$. ambatomisana n. sp. Ce groupe est caractérisé par des lobes latéraux du dixième segment abdominal spiniformes et allongés et des appendices supérieurs absents ou vestigiaux ( $R$. rakotonirinai n. sp.). Chez $R$. humberti n. sp., la carène dorsale du lobe médian est longue ce qui donne une forme patatoïde à la vue latérale du dixième segment abdominal. Elle est, en revanche, courte chez 

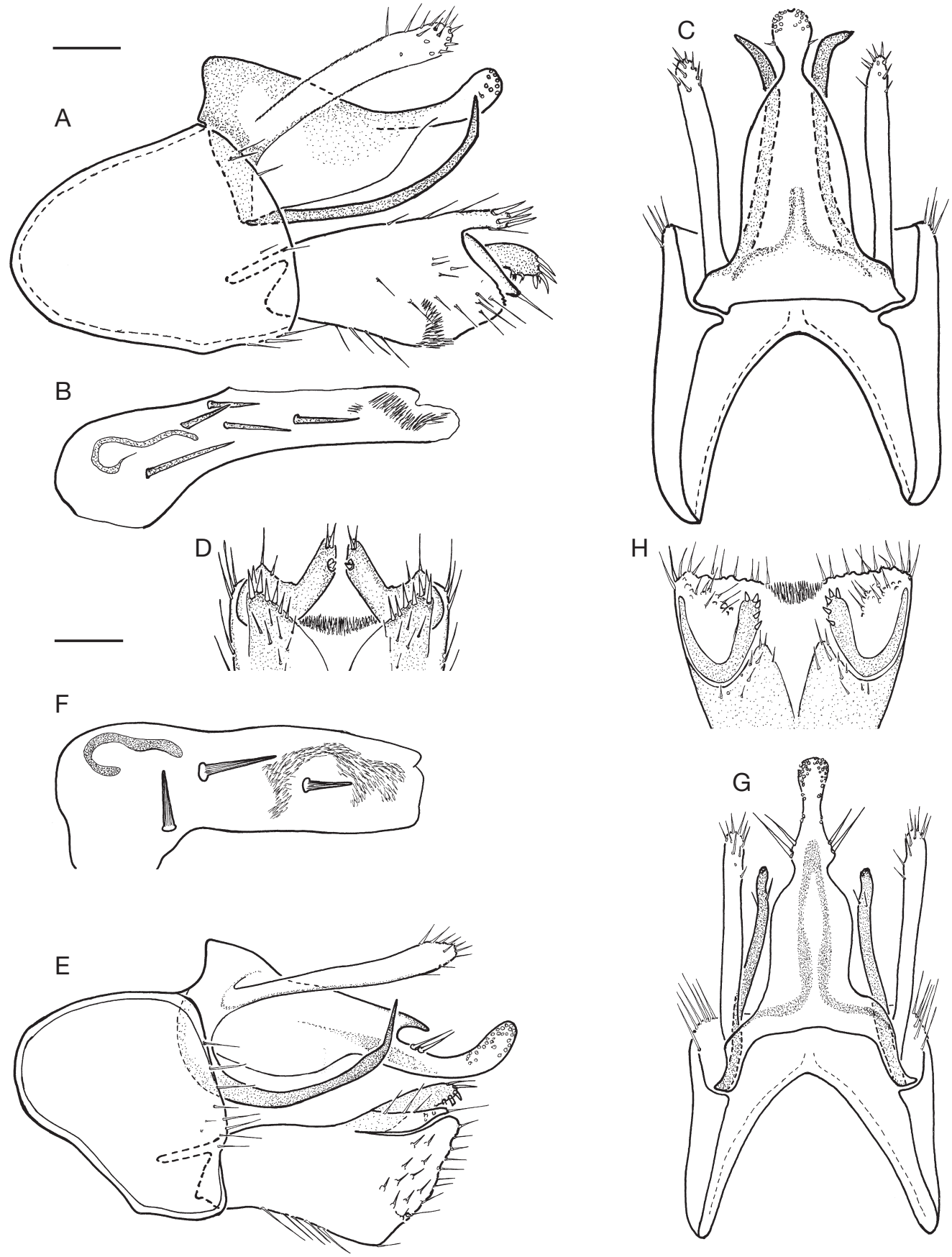

FIG. 6. - A-D, Rossodes namorona n. sp. ; A, vue latérale des genitalia ; B, vue latérale de l'appareil phallique ; C, vue dorsale du dixième tergite ; $\mathbf{D}$, vue dorsale partielle des appendices inférieurs ; E-H, $R$. marojejyensis n. sp. ; E, vue latérale des genitalia ; F, vue latérale de l'appareil phallique; $\mathbf{G}$, vue dorsale du dixième tergite et du neuvième segment ; $\mathbf{H}$, vue dorsale partielle des appendices inférieurs. Échelles : 0,1 mm. 
R. rakotonirinai n. sp. et $R$. ambatomisana n. sp., ce qui donne une forme triangulaire à la vue latérale du dixième segment abdominal. Chez $R$. humberti n. sp., on note également la présence de deux paires d'épines internes à l'appareil phallique, lequel comporte également un sclérite phallotrémal ( $R$. rakotonirinai n. sp. et $R$. ambatomisana n. sp. ne possèdent qu'une seule paire de sclérites internes à l'appareil phallique).

\section{DESCRIPTION}

Taille. Aile antérieure : $0,59 \mathrm{~cm}$, aile postérieure : $0,53 \mathrm{~cm}$.

Genitalia mâles. Neuvième tergite fusionné avec la base du dixième. Appendices inférieurs : structure caractéristique du genre ; second article court épais et légèrement courbé (vue dorsale). Appendices supérieurs disparus ou fusionnés au dixième tergite. Extrémités antérieures du dixième tergite déformées en un long lobe spiniforme, droit, en forme d'épieu, presque indépendant. Élément central du dixième tergite large et massif tant en vue latérale que dorsale ; une carène dorsale longue ; extrémité arrondie en vue latérale, presque droite en vue dorsale. Appareil phallique muni d'un sclérite phallotrémal, d'une petite zone de spicules et de deux paires d'épines internes.

\section{Rossodes rakotonirinai $\mathrm{n}$. $\mathrm{sp}$.}

(Fig. 8A-D)

MATÉRIEL TYPE. - Holotype : Parc national de l'Andohahela, 18.V.1994, F.-M. Gibon, F. Ranaivoharindriaka, $10^{\prime \prime}(\mathrm{MNHN})$.

LOCALITÉ TYPE. - Massif d'Andohahela, sur l'Ambahibe (Bassin de la Efaho, 46⒌'07”E, 2446'17”S)

Distribution. - La distribution connue se limite au massif d'Andohahela.

ÉTYMologie. - Cette espèce est dédiée à la mémoire de Jean-Bernard Rakotonirina, chauffeur au LRSAE.

DiAGNOSE. - Cette espèce est très proche de $R$ ambatomisana n. sp. Elles présentent toutes deux des lobes latéraux du dixième segment abdominal allongés et spiniformes; elles se distinguent de $R$. langrandi n. sp. par l'absence d'appendices supérieurs et de $R$. humberti n. sp. par la présence d'une seule paire de sclérites internes à l'appareil phallique ainsi que par la forme triangulaire du lobe médian du dixième segment. Rossodes rakotonirinai $\mathrm{n}$. sp. se distingue de R. ambatomisana n. sp. par la forme des appendices inférieurs, dont l'angle dorso-distal est peu développé, laissant apparaître le deuxième segment (vue latérale), ainsi que par la forme de l'appareil phallique mince et offrant, en vue latérale, une courbure régulière.

\section{DESCRIPTION}

Taille. Aile antérieure : $0,48 \mathrm{~cm}$, aile postérieure : $0,41 \mathrm{~cm}$.

Genitalia mâles. Neuvième tergite fusionné avec la base du dixième. Appendices inférieurs : structure caractéristique du genre; second article court épais et courbé (vue dorsale). Appendices supérieurs disparus ou fusionnés au dixième tergite. Extrémités antérieures du dixième tergite déformées en un long lobe spiniforme distal, droit, en forme d'épieu légèrement courbé dorsalement. Élément central du dixième tergite large et grossièrement triangulaire en vue latérale, bosse sommitale prononcée; en vue dorsale, base large rapidement amincie, nombreuses sensillae distales. Appareil phallique allongé muni d'une seule paire de sclérites internes en forme de C dont la branche dorsale allongée (vue latérale).

\section{Rossodes ambatomisana n. sp.} (Fig. 8E-H)

MATÉRIel TYPe. - Holotype : Région d'Anjozorobe, 8.XI.1995, 1 ơ (MNHN).

Paratypes : Même localité que l'holotype, 18.X.1995, $20^{7} 0^{7}$; deux ơ $^{7}$, 31.X.1996 à proximité de la localité de l'holotype sur un petit ruisseau forestier (Bassin de la Betsiboka, 4756 $\left.53^{\prime \prime} \mathrm{E}, 18^{\circ} 27^{\prime} 13^{\prime \prime} \mathrm{S}\right)$ (CBGP).

LOCALITÉ TYPE. - Au nord d'Antananarivo sur l'Ambatomisana, à la lisière entre la forêt et les plantations de géranium (Bassin de la Betsiboka, 4757’07”'E, $18^{\circ} 27^{\prime} 15^{\prime \prime}$ ).

DisTRIBUTION. - La distribution connue se limite aux environs de la localité type, dans des forêts humides primaires au nord est d'Antananarivo.

ÉTYMOLOGIE. - Cette espèce est nommée en référence à la rivière Ambatomisana, où elle a été découverte.

DiAGNOSE. - Rossodes ambatomisana $\mathrm{n}$. sp. est très proche de $R$. rakotonirinai $\mathrm{n}$. sp. Les deux espèces présentent des lobes latéraux du dixième segment abdominal allongés et spiniformes. Elles se distinguent de R. langrandi n. sp. par l'absence d'appendices supérieurs et de $R$. humberti n. sp. 


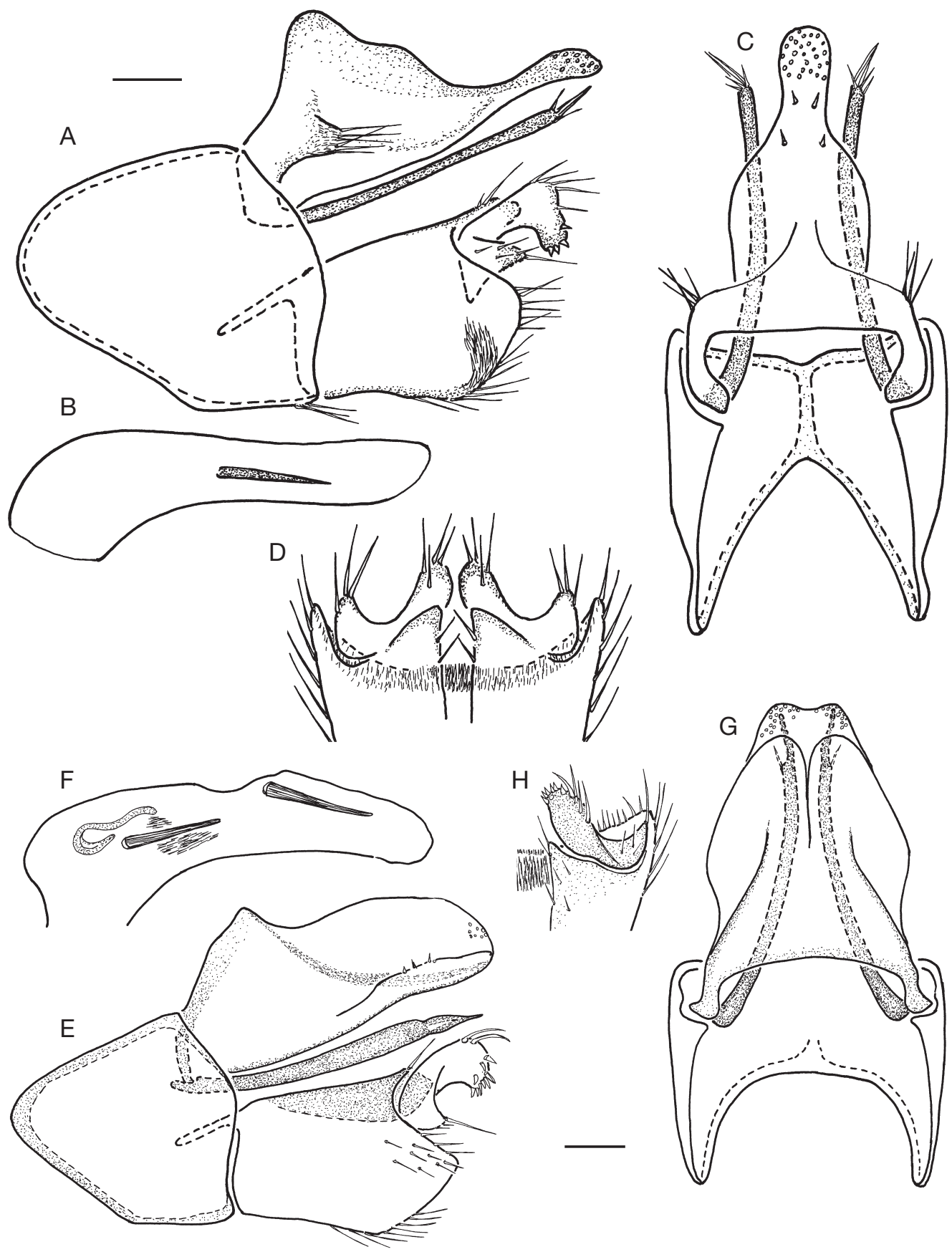

FIG. 7. - A-D, Rossodes langrandi n. sp. ; A, vue latérale des genitalia ; B, vue latérale de l'appareil phallique ; C, vue dorsale du dixième tergite ; $\mathbf{D}$, vue dorsale partielle des appendices inférieurs; $\mathbf{E}-\mathbf{H}, R$. humberti n. $\mathrm{sp}$. ; $\mathbf{E}$, vue latérale des genitalia ; $\mathbf{F}$, vue latérale de l'appareil phallique; $\mathbf{G}$, vue dorsale du dixième tergite et du neuvième segment ; $\mathbf{H}$, vue dorsale partielle des appendices inférieurs. Échelles : 0,1 mm. 
par la présence d'une seule paire de sclérites internes à l'appareil phallique ainsi que par la forme triangulaire du lobe médian du dixième segment. Rossodes ambatomisana n. sp. se distingue de $R$. rakotonirinai $\mathrm{n}$. sp. par la forme des appendices inférieurs, dont l'angle dorso-distal, très développé, masque complètement le deuxième segment (vue latérale). L'espèce est également caractérisée par la forme de l'appareil phallique, qui offre un léger renflement médian et dont la face dorsale est plus longuement sclérifiée que la ventrale.

\section{DESCRIPTION}

Taille. Aile antérieure : $0,53 \mathrm{~cm}$, aile postérieure : $0,47 \mathrm{~cm}$.

Genitalia mâles. Neuvième tergite fusionné avec la base du dixième. Appendices inférieurs : structure caractéristique du genre; second article à base très large. Appendices supérieurs disparus ou fusionnés au dixième tergite. Aux angles proximo-ventraux du dixième tergite apparaît un long lobe spiniforme, droit, en forme d'épieu légèrement courbé dorsalement, il constitue un sclérite quasiment indépendant de la partie centrale du tergite; cette dernière est large en vue latérale avec une bosse dorsale prononcée. Appareil phallique allongé muni d'une paire de sclérites internes caractérisés par une base courbée et une partie distale spiniforme.

\section{Rossodes ankaratra n. sp.}

(Fig. 9A-D)

MATÉRIEL TYPE. - Holotype: 1 ơ (MNHN), 8.III.1995, capturé au piège lumineux.

LOCALITÉ TYPE. - Massif de l'Ankaratra, sur un petit tributaire du Mangoro (47¹9'11'E, 19'21'00”S).

DisTRIBUTION. - La distribution connue se limite à la localité-type : une petite zone boisée relictuelle sur les hauteurs de l'Ankaratra.

ÉTYMOLOGIE. - Cette espèce est nommée en référence au massif de l'Ankaratra où elle a été découverte.

DiAgnOSE. - Rossodes ankaratra n. sp. est caractérisé par l'épaisseur et la longueur des éléments latéraux du dixième segment, ainsi que par la taille des soies qu'ils portent. Ces caractères empêchent toute confusion avec une autre espèce. On notera, également, en vue dorsale, l'invagination à l'extrémité du lobe médian. Ce caractère ne se retrouve que chez $R$. goodmani $\mathrm{n}$. sp. dont les lobes latéraux sont beaucoup plus courts et en forme de dague (vue latérale).

\section{DESCRIPTION}

Taille. Aile antérieure : $0,62 \mathrm{~cm}$, aile postérieure : $0,55 \mathrm{~cm}$

Genitalia mâles. Neuvième tergite fusionné avec la base du dixième. Appendices inférieurs : structure caractéristique du genre. Appendices supérieurs disparus ou fusionnés au dixième tergite. Angles proximo-ventraux du dixième tergite déformés en un long lobe très sclérifié, en forme de grosse branche dirigée distalement et dont le bord supérieur porte une série de fortes soies. Élément central du tergite triangulaire en vue latérale, légèrement et régulièrement amincie en vue dorsale, une invagination en à l'extrémité distale. Appareil phallique muni d'un sclérite phallotrémal et de huit paires d'épines internes, sept assez courtes et de forme typique, la huitième plus longue, à l'aspect torsadé.

\section{ROSSODINAE : RÉÉVALUATION DES CARACTÈRES DE LA SOUS-FAMILLE}

La découverte d'un grand nombre d'espèces conduit à revoir plus ou moins profondément les diagnoses antérieures. En effet, lorsque Ross (1956) décrit une sous-famille, un genre et une espèce à partir de deux individus probablement conspécifiques, il ne peut avec certitude attribuer les caractères utilisés à un niveau taxonomique donné. Pour caractériser les Rossodinae, il se fonde sur la combinaison des caractères suivants : la présence de la furca 4 aux ailes postérieures du mâle, la présence de trois nervures anales non modifiées aux ailes postérieures, la forme annulaire du neuvième segment abdominal et la structure modifiée des appendices inférieurs. Chez les Philopotamidae, les trois premiers caractères sont plésiomorphes. Le quatrième (la structure particulière des appendices inférieurs) était caractéristique de $R$. tsaratananae; nous retrouvons cette structure, avec des variations morphologiques mineures, chez toutes les nouvelles espèces. Il s'agit donc d'une apomorphie qui atteste la monophylie de la lignée. Une conclusion particulièrement utile, car Rossodes est maintenant bien moins homogène en 

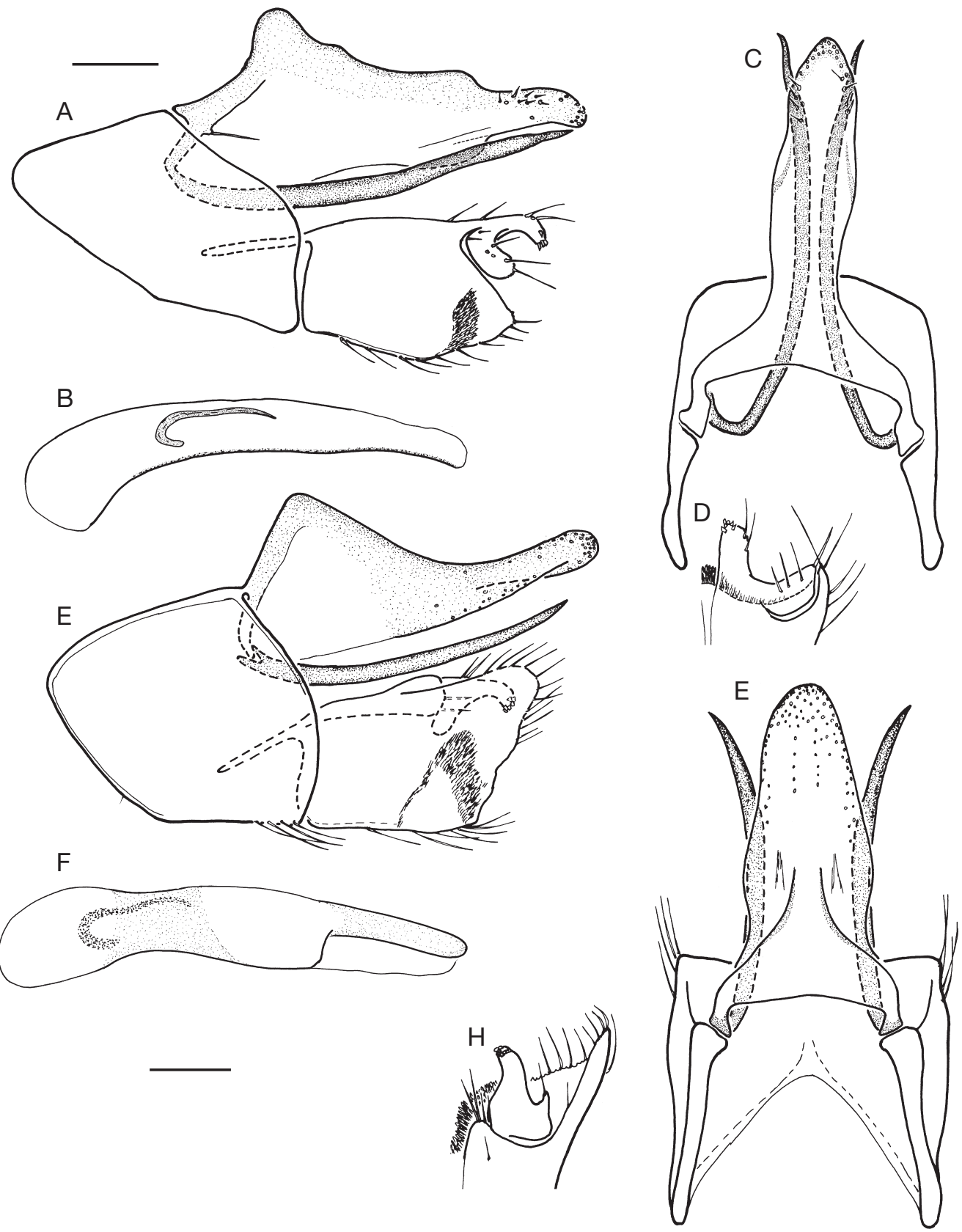

FIG. 8. - A-D, Rossodes rakotonirinai n. sp. ; A, vue latérale des genitalia ; B, vue latérale de l'appareil phallique ; C, vue dorsale du dixième tergite ; D, vue dorsale partielle des appendices inférieurs ; E-H, $R$. ambatomisana n. sp.; $\mathbf{E}$, vue latérale des genitalia ; $\mathbf{F}$, vue latérale de l'appareil phallique; $\mathbf{G}$, vue dorsale du dixième tergite et du neuvième segment ; $\mathbf{H}$, vue dorsale partielle des appendices inférieurs. Échelles : 0,1 mm. 
ce qui concerne les trois autres caractères, qui sont parfois très modifiés.

La présence de trois nervures anales non modifiées aux ailes postérieures est observée chez toutes les nouvelles espèces. C'est la principale caractéristique de ce que Ross (1956) a nommé le " complexe primitif ", un groupe composé des Rossodinae et des Philopotaminae à l'exception du genre Wormaldia McLachlan, 1865. Elle distingue ce complexe des Chimarra Stephens, 1829 et des Wormaldia chez lesquels la nervure A2 fusionne ou rejoint A1.

La présence de la furca 4 aux ailes postérieures du mâle de $R$. tsaratananae est une caractéristique remarquable, puisqu'elle est absente chez tous les autres Philopotamidae. Mais, d'une part, elle est absente chez la femelle (le deuxième spécimen étudié par Ross), d'autre part, elle est absente chez toutes les nouvelles espèces. Elle n'a donc été observée que sur un individu, le seul mâle connu de $R$. tsaratananae. Ceci mériterait une confirmation car la nervation présente parfois des irrégularités, bien que celles-ci affectent rarement les deux ailes d'une manière symétrique.

La présence d'un neuvième tergite sclérifié et indépendant du dixième constitue un caractère qui distingue $R$. tsaratananae des autres lignées du complexe primitif sensu Ross, c'est-à-dire qu'il distingue les Rossodinae des Philopotaminae. Nous le retrouvons chez quatre des 14 nouvelles espèces ( $R$. mantadia n. sp., $R$. pilakai n. sp., $R$. goodmani n. sp., $R$. hertui n. sp.). Chez $R$. fabienneae n. sp., le neuvième tergite est réduit à une mince bande faiblement sclérifiée, alors que chez $R$. manantenina n. sp. il est partiellement ouvert dorsalement. Chez les autres espèces, il est absent ou a fusionné avec le dixième tergite (ce qui se traduit par le fait que l'on ne puisse pas observer de séparation entre ces deux tergites).

Ross décrit les appendices supérieurs comme des « expansions latérales de la base du dixième tergite "; il y a donc, à l'extrémité de l'abdomen de $R$. tsaratananae, un ensemble constitué d'un élément central massif (le dixième tergite) portant deux éléments latéraux moins sclérifiés (les appendices supérieurs). Cette structure se retrouve chez toutes les nouvelles espèces. Mais, la plupart d'entre elles présentent d'importantes modifications de la base du dixième tergite. Les extrémités latérales antérieures se retournent et se prolongent distalement en un lobe sclérifié qui prend naissance sous l'appendice supérieur. C'est le cas chez $R$. marojejyensis n. sp. où l'appendice supérieur a une forme en massue portant un groupe de soies terminales et le prolongement distal du bord antérieur, plus sclérifié, une forme en épieu courbé dorsalement (Fig. 6E). Chez $R$. langrandi n. sp., la structure est identique mais l'appendice supérieur est réduit à une bosse couverte de longues soies (Fig. 7A). Cette réduction de l'appendice supérieur chez $R$. langrandi n. sp., permet d'interpréter les structures présentes chez $R$. humberti n. sp., $R$. rakotonirinai n. sp. et $R$. ambatomisana n. sp. comme résultant d'une disparition de l'appendice supérieur et de la formation de deux lobes latéraux en forme d'épieu; ces derniers sont presque indépendants du lobe central chez $R$. humberti n. sp. et $R$. ambatomisana n. sp. (Figs 7E, 8E). Chez d'autres espèces, l'interprétation est plus délicate : il est probable qu'il y ait une fusion entre l'appendice supérieur et une partie du sclérite. Il en résulte un élément latéral qui présente une partie couverte de petites soies et une partie plus sclérifiée souvent en forme de poignard (Fig. 3A, 2E). La diversité de ces structures rend délicate l'interprétation chez $R$. ankaratra n. sp. En raison d'une forte proximité avec $R$. rakotonirinai n. sp., notamment dans la structure du neuvième segment, nous interprétons l'extrémité de l'abdomen comme un dixième tergite constitué d'un lobe médian massif encadré de deux lobes latéraux en forme de grosse branche. Il nous faut alors supposer la disparition des appendices supérieurs, ou leur fusion avec les lobes latéraux. Cette interprétation est cohérente avec l'ensemble des espèces dont nous disposons aujourd'hui, elle pourrait être modifiée par la découverte de nouvelles espèces présentant d'autres tendances évolutives.

\section{GÉOGRAPHIE ET ÉCOLOGIE}

La répartition géographique des Rossodinae se limite à la Grande Île. C'est l'un des nombreux taxons qui font l'originalité de la faune malgache et justifient les efforts entrepris pour sa conservation. L'étude de leur répartition sur l'île apporte quelques éléments remarquables. Le genre Rossodes est présent depuis la Montagne d'Ambre jusqu'au massif d'Andohahela, 
c'est-à-dire du nord au sud de Madagascar (Fig. 10). Nous l'avons capturé depuis le niveau de la mer jusqu'à des altitudes de 1700 et $1800 \mathrm{~m}$ sur les massifs de l'Andringitra et de l'Ankaratra (Fig. 11). L'existence de limites altitudinales de distribution est, en revanche, très probable au niveau spécifique. Ainsi sur le Marojejy, où quatre étages altitudinaux ont été échantillonnés, $R$. manantenina $\mathrm{n}$. sp. se rencontre dans la zone des $450 \mathrm{~m}$ et dans celle des $750 \mathrm{~m}, R$. humberti n. sp. dans celles des 750 et $1200 \mathrm{~m}$, enfin $R$. marojejyensis n. sp. dans celles des 1200 et $1600 \mathrm{~m}$. Autre exemple à la latitude d'Antananarivo : $R$. ankaratra n. sp. est capturé vers $1800 \mathrm{~m}$ sur l'Ankaratra, $R$ ambatomisana n. sp. de 1200 à $1300 \mathrm{~m}$ dans la région d'Anjozorobe et $R$. mantadia n. sp. à $950 \mathrm{~m}$ au nord d'Andasibe. Des études régionales devraient confirmer ces premières observations. Comme les autres Philopotamidae, Rossodes se rencontre dans les eaux courantes, mais uniquement sur de petits, voire très petits, cours d'eau, généralement moins de $5 \mathrm{~m}$ de largeur (Fig. 11). Par ailleurs, aucune capture n'a été réalisée hors des forêts humides primaires. La présence et l'intégrité de cette végétation sur le bassin versant sont nécessaires au développement des Rossodes. Il faut souligner qu'il s'agit bien de la végétation du bassin versant et pas seulement de la végétation rivulaire. Il est possible que les larves soient particulièrement sensibles à la turbidité et aux charges sédimentaires, mais les liens entre la végétation terrestre, les caractéristiques physico-chimiques des eaux et les conditions benthiques sont si nombreuses qu'il est illusoire de rechercher un déterminisme simple. À ce sujet, il est intéressant à noter que Ross lui-même (1963) a été l'un des premiers à avoir mis en évidence les effets du biome sur la répartition des insectes aquatiques. Ce n'est pas un hasard : la répartition des Trichoptères rhithriques, qui ont constitué le principal sujet de ses travaux, se révèle souvent dépendante des formes de végétation du bassin versant. Ces travaux concernaient l'Amérique du Nord; les données tropicales étaient trop ponctuelles. Depuis, ce type de relation a été mis en évidence en Afrique occidentale (Quillévéré \& Pendriez 1975 ; Quillévéré et al. 1981 ; Gibon \& Statzner 1985 ; Gibon 2001).
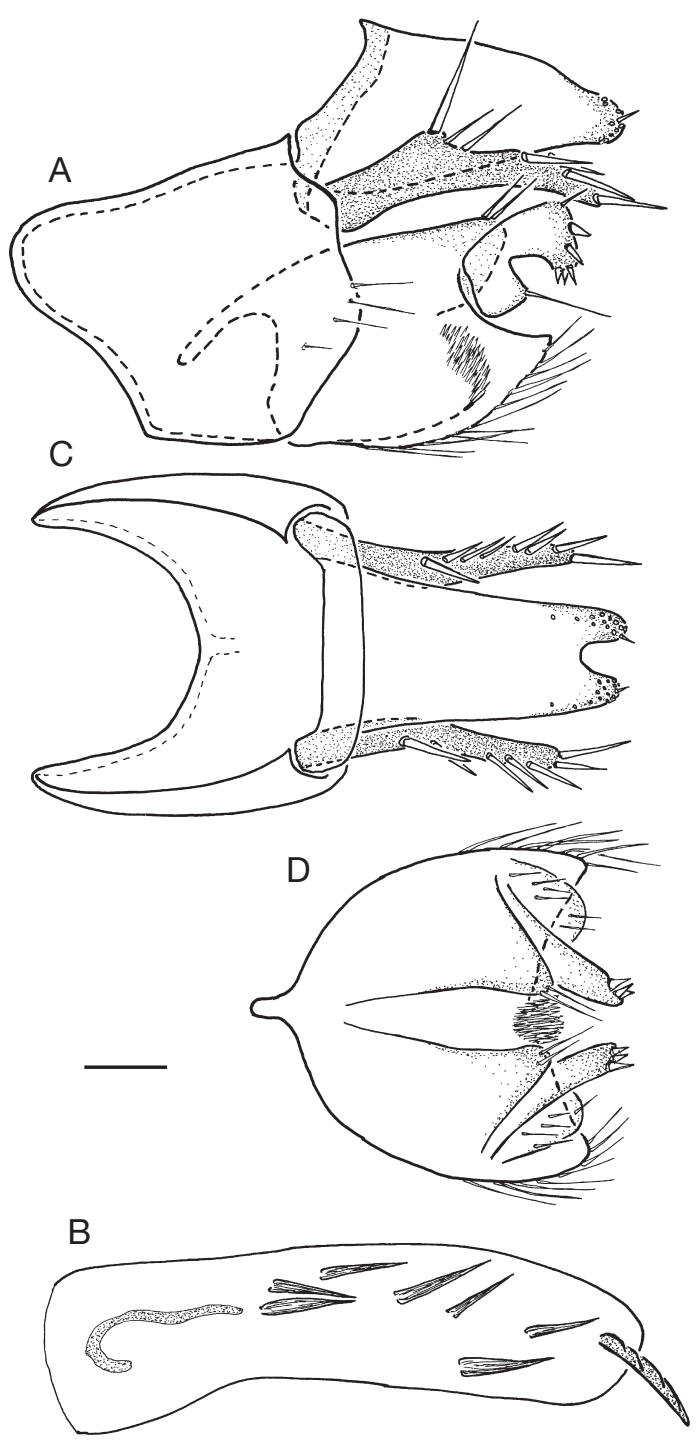

FIG. 9. - A-D, Rossodes ankaratra n. sp. ; A, vue latérale des genitalia ; B, vue latérale de l'appareil phallique ; C, vue dorsale du dixième tergite; $\mathbf{D}$, vue dorsale partielle des appendices inférieurs. Échelle : 0,1 mm.

Si le genre Rossodes est présent dans la plupart des forêts humides primaires, les aires de répartition des espèces sont, en revanche, réduites. Aucune espèce n'est présente dans deux ou plus des zones d'échantillonnage recensées (Fig. 10). Ainsi les espèces de la zone 1 (Parc national de la Montagne 


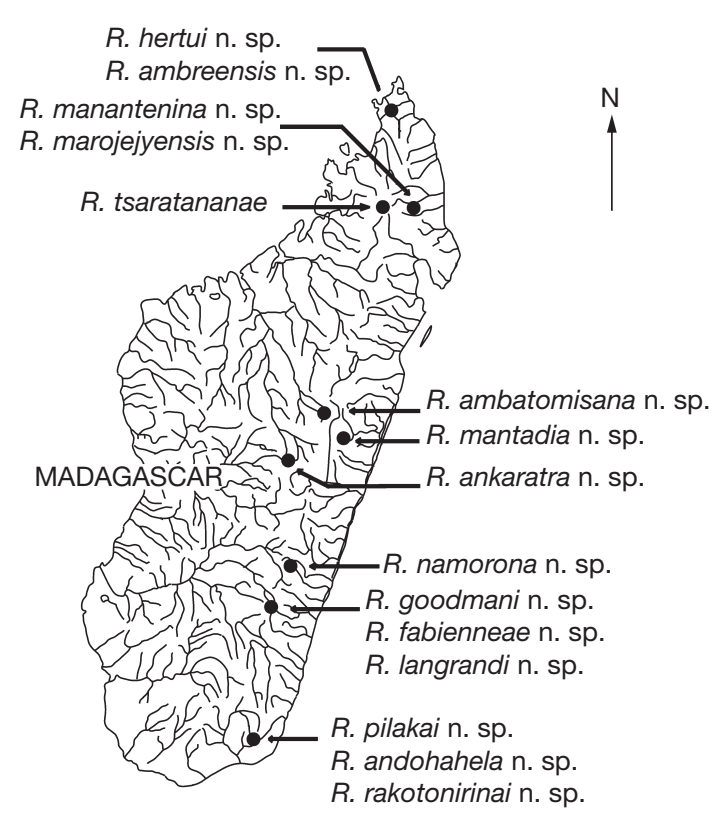

FIG. 10. - Carte des sites de captures des espèces du genre Rossodes Özdikmen \& Darilmaz, 2008.

d'Ambre) sont $R$. hertui n. sp. et $R$. ambreensis n. sp., celles de zone 2 (Parc national du Marojejy) sont $R$. manantenina n. sp., $R$. marojejyensis $\mathrm{n}$. sp. et $R$. humberti n. sp., celle du point 3 (Massif du Tsaratanana, localité-type du matériel capturé par R. Paulian et ses collaborateurs puis étudié par Ross) est $R$. tsaratanana, celle de la zone 4 (Forêt d'Anjozorobe) est $R$. ambatomisana n. sp., celle de la zone 5 (Parc national de Mantadia) est $R$. mantadia n. sp., celle de la zone 6 (Réserve naturelle de l'Ankaratra) est $R$. ankaratra n. sp., celle de la zone 7 (Parc national de Ranomafana) est $R$. namorona n. sp., celles de la zone 8 (Parc national de l'Andringitra) sont $R$. goodmani n. sp., $R$. fabienneae n. sp. et $R$. langrandi n. sp., celles de la zone 9 (Parc national d'Andohahela) sont $R$. pilakai n. sp., $R$. andohahela n. sp. et $R$. rakotonirinai n. sp. Ce morcellement en espèces à petites aires de distribution s'explique par la combinaison d'un facteur écologique, la forêt, et d'un facteur historique, le refroidissement au cours des dernières périodes glaciaires. D'un point de vue écologique, la forêt s'oppose au vol et à la dispersion des adultes, elle

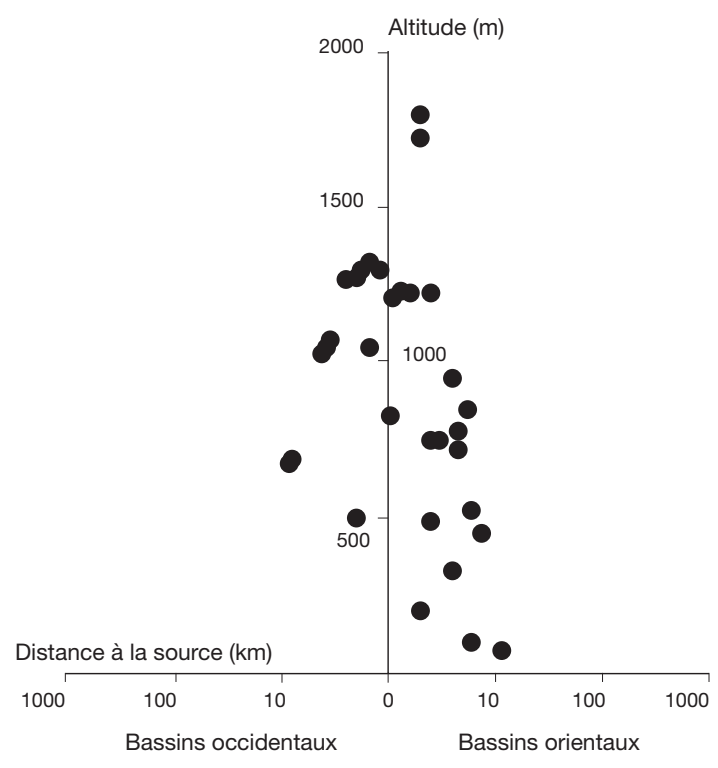

FIG. 11. - Profil écologique des sites de captures du genre Rossodes.

favorise plus la spéciation locale que les espaces ouverts et les vents associés. De plus, steppes et savanes correspondent à des climats plus secs où, en l'absence de formes de résistance, de nombreux petits cours d'eau sont recolonisés par des adultes après les sécheresses saisonnières ou accidentelles, un phénomène qui nécessite de bonnes capacités de vol et constitue un facteur d'homogénéisation génétique des populations.

Au cours des dernières glaciations, la végétation malgache de haute montagne, une brousse à Ericaceae, s'est considérablement étendue (Burney 1996), jusqu'à descendre sous les $900 \mathrm{~m}$ dans la région de Moramanga (Rossi 1978). Les forêts humides se sont alors retrouvées morcelées en plusieurs zones refuges sur la côte orientale et dans quelques grandes vallées septentrionales (Straka 1996). Ces refuges sont probablement à l'origine des diverses espèces qui se succèdent du nord au sud de la zone des forêts humides orientales. Les variations climatiques quaternaires ont été utilisées récemment pour expliquer le microendémisme malgache chez les vertébrés (Wilmé et al. 2006). Pour les populations de Rossodes, plus généralement pour la faune des petits torrents montagneux, le 
phénomène a été accentué par le fait que les vallées et les zones marécageuses ont constitué des barrières supplémentaires.

\section{Remerciements}

Je tiens à remercier toute l'équipe du Laboratoire de Recherches sur les Systèmes Aquatiques et leur Environnement notamment Jean-Marc Elouard, Désiré Randriamasimanana, Jean-Luc Gattolliat, Abel Ralaiteferana, Zoé Andriambelo, Théogène Pilaka, qui ont participé aux captures et, naturellement, tous ceux dont j'ai utilisé le patronyme ou le prénom pour nommer les espèces. Ma gratitude va également aux trois relecteurs de Zoosytema, Joël Minet, Henri Tachet et Annemarie Ohler, dont les remarques ont permis d'améliorer ce travail.

\section{RÉFÉRENCES}

ANONYME 1970. - Nouvelles données scientifiques sur le massif du Tsaratanana (Madagascar) : résultats de la mission concertée de novembre 1966 : armée française-ORSTOM. Mémoire ORSTOM n³7, ORSTOM, Paris, $251 \mathrm{p}$.

BLAHNIK R. J. 2002. - Systematics of Otarrha, a new Neotropical subgenus of Chimarra (Trichoptera: Philopotamidae). Systematic Entomology 27: 65130.

BLAHNIK R. J. 2005. - Alterosa, a new caddisfly genus from Brazil (Trichoptera: Philopotamidae). Zootaxa 991: 3-60.

BuRnEY D. A. 1996. - Climate change and fire ecology as factors in the quaternary biogeography of Madagascar, in Lourenço W. R. (éd.), Biogéographie de Madagascar. ORSTOM, Paris: 49-58.

ElOUARD J.-M. \& GibOn F.-M. 2001. — Biodiversité et biotypologie des eaux continentales de Madagascar. IRD, Montpellier, $447 \mathrm{p}$.

GiBON F.-M. 2000. - Biologie de la conservation et singularité des cours d'eau. L'exemple des Philopotamidae malgaches (Insecta, Trichoptera), in Lourenço W. R. \& Goodman S. M. (eds), Diversity and Endemism in Madagascar. Société de Biogéographie, Paris: 319-330.

GiBON F.-M. 2001. - Biotypologie des hydrosystèmes tropicaux, exemples chez les Trichoptères africains et malgaches. IRD, Montpellier, $381 \mathrm{p}$.

Gibon F.-M. 2004. - Trichoptera, Caddisflies, in Goodman S. M. \& Benstead J. P. (éds), The Natural History of Madagascar. The University of Chicago Press, Chicago: 740-748.
Gibon F.-M. \& STATZner B. 1985. - Longitudinal zonation of lotic insects in the Bandama River System (Ivory Coast). Hydrobiologia 122: 61-64.

GibON F.-M. \& ElouarD J.-M. 1996. - Étude préliminaire de la distribution des insectes lotiques à Madagascar (exemples des Trichoptères Philopotamidae et Diptères Simuliidae), in W.R. Lourenço (ed.), Biogéographie de Madagascar, ORSTOM, Paris: 507-516.

GibON F.-M. \& ANDRIAMBELo P. Z. 1999. — A regional analysis of species associations and distributions of two caddisflies families in the South-East of Madagascar (Aquatic Biodiversity of Madagascar. 10), in Goodman, S.M. (ed.), A floral and faunal inventory of the Réserve Naturelle Intégrale d'Andohahela, Madagascar : With reference to elevational variation. Fieldiana (Zoology) N.S. 94: 97-109

GibOn F.-M., Elouard J.-M. \& SARTORI M. 1996. Spatial Distribution of Some Aquatic Insects in the Réserve Naturelle Intégrale d'Andringitra, Madagascar, in Goodman, S.M. (ed.), A floral and faunal inventory of the eastern slopes of the Réserve Naturelle Intégrale d'Andringitra, Madagascar: With reference to elevational variation. Fieldiana (Zoology) N.S. 85: 109-120.

Gibon F.-M., ANdriambelo P. Z. \& RandriamasimaNANA D. 1999. - A study of the diversity and richness of the Malagasy Trichoptera, in Chantaramongkol P. \& Malicky H. (eds), Proceedings of the 9th International Symposium on Trichoptera. Université de Chiang-Maï, Thaillande: 123-124.

GoOdman S. 1996. - A floral and faunal inventory of the eastern slopes of the réserve naturelle intégrale d'Andringitra, Madagascar: with reference to elevational variation. Fieldiana: Zoology (new series) 85: 1-319.

GOODMAN S. 1998. - A floral and faunal inventory of the réserve spéciale d'Anjanaharibe-Sud, Madagascar: with reference to elevational variation. Fieldiana: Zoology (new series) 90: 1-246.

GOODMAN S. 1999. - A floral and faunal inventory of the réserve naturelle intégrale d'Andohahela, Madagascar: with reference to elevational variation. Fieldiana: Zoology (new series) 94: 1-297.

GOODMAN S. 2000. - A floral and faunal inventory of the Parc national de Marojejy, Madagascar: with reference to elevational variation. Fieldiana: Zoology (new series) 97: 1-286.

Holzenthal R. W., Blahnik R. J., Prather A. L. \& KJER, K. M. 2007. - Order Trichoptera Kirby, 1813 (Insecta), caddisflies. Zootaxa 1668: 639-698.

KJer K. M., Blahnik R. J. \& Holzenthal R. W. 2002. - Phylogeny of caddisflies (Insecta, Trichoptera). Zoologica Scripta 31: 83-91. Mamet J. R. 1954. - Notes on the Coccoidea of Madagascar, III. Mémoires de l'Institut Scientifique de Madagascar (Série E) 4: 1-86.

MunOZ-Quesada F. J. \& Holzenthal R. W. 2008. — 
Revision of the Nearctic species of the caddisfly genus Wormaldia McLachlan (Trichoptera: Philopotamidae). Zootaxa 1838: 1-75.

ÖZDIKMEN H. \& DariLMAZ M. 2008. — New subfamily and genus names, Rossodinae nom. nov. and Rossodes nom. nov., for the finger-net Caddisflies (Trichoptera: Philopotamidae). Munis Entomology and Zoology 3 (1): 162-164.

Quillévéré D. \& Pendriez B. 1975. — Étude du complexe Simulium damnosum en Afrique de l'Ouest : 2. Répartition géographique des cytotypes en Côte d'Ivoire. Cahiers ORSTOM, Série Entomologie Médicale et Parasitologie 13 (3): 85-98.

Quillévéré D., Guillet P. \& Séchan Y. 1981. — La répartition géographique des espèces du complexe Simulium damnosum dans la zone du projet Sénégambie. Cahiers ORSTOM, Série Entomologie Médicale et Parasitologie 19 (4): 303-311.

RANDRIAMASIMANANA D. \& GIBON F.-M. 2001. —Étude systématique, habitats et répartition géographique des
Setodes (Trichoptera : Leptoceridae) de Madagascar. Annales de Limnologie 37: 125-141.

Ross H. H. 1956. - Evolution and Classification of the Mountain Caddisflies. University of Illinois Press, Urbana, 213 p.

Ross H. H. 1963. - Stream communities and terrestrial biomes. Archiv für Hydrobiologie 59: 235-242.

Rossi G. 1978. - Sur l'extension de la phase aride Pliocène dans le massif du Tsaratanana. Revue de Géographie (Madagascar) 33: 69-76.

SCHMID F. 1998. - Genera of the Trichoptera of Canada and adjoining or adjacent United States. National Research Council of Canada, Ottawa: $319 \mathrm{p}$.

STRAKa H. 1996. - Histoire de la végétation de Madagascar oriental dans les derniers dix millénaires, in LOURENÇO W. R. (éd.), Biogéographie de Madagascar. ORSTOM, Paris: 37-47.

Wilmé L., GoOdman S. M. \& GANZHORN J. U. 2006. Biogeographic evolution of Madagascar's microendemic biota. Science 312: 1063-1065.

Soumis le 21 juin 2012; accepté le 25 février 2013 ; publiéle 28 juin 2013. 